\title{
GALOIS THEORY OF FUCHSIAN $q$-DIFFERENCE EQUATIONS
}

\author{
BY JACQUES SAULOY
}

ABSTRACT. - We propose an analytical approach to the Galois theory of singular regular linear $q$-difference systems. We use Tannaka duality along with Birkhoff's classification scheme with the connection matrix to define and describe their Galois groups. Then we describe fundamental subgroups that give rise to a Riemann-Hilbert correspondence and to a density theorem of Schlesinger's type.

(C) 2003 Elsevier SAS

RÉSUMÉ. - Nous proposons une approche analytique de la théorie de Galois des systèmes aux $q$-différences linéaires singuliers réguliers. Nous combinons la dualité de Tannaka avec la méthode de classification de Birkhoff à l'aide de la matrice de connexion pour définir et décrire leurs groupes de Galois. Puis nous décrivons des sous-groupes fondamentaux qui donnent lieu à une correspondance de RiemannHilbert et à un théorème de densité de type Schlesinger.

(c) 2003 Elsevier SAS

"Je suis convaincu que, tout comme pour les fonctions spéciales solutions d'équations différentielles, les formules intéressantes dérivent de considérations "géométriques" simples" (Jean-Pierre Ramis, [23]).

\section{Introduction}

\subsection{Rational linear $q$-difference systems and rational equivalence}

Let $q$ be a fixed complex number such that $|q|>1$. Let $\sigma_{q}$ denote the dilatation $z \mapsto q z$ of a complex coordinate $z$ on the Riemann sphere $\mathbf{S}=\mathbf{P}^{1} \mathbf{C}$, viewed as an operator on functions of $z$. In [27], we studied the classification of rational linear q-difference systems

$$
\sigma_{q} X=A X
$$

over $\mathbf{S}$ under rational equivalence. Here, $A \in G L_{n}(\mathbf{C}(z))$ is a given matrix and the unknown $X$ is a matrix with $n$ rows and (possibly multivalued) holomorphic entries on some $q$-invariant open subset of $\mathbf{S}$. The gauge group $G L_{n}(\mathbf{C}(z))$ operates on the left on solutions of such systems, hence on the systems themselves. This gives rise to the rational equivalence relation:

$$
A \sim\left(\sigma_{q} F\right)^{-1} A F, \quad F \in G L_{n}(\mathbf{C}(z)) .
$$

In this paper, we study the Galois theory of such systems. 


\subsection{The classification theorem of Birkhoff}

In analogy with Riemann's classification scheme for complex fuchsian differential equations, by local data at the singularities and monodromy transformations arising from the analytic continuation of local solutions (the so-called "connection formulae"), Birkhoff defined in [5] the "generalized Riemann problem" for $q$-difference equations. He solved it for fuchsian systems under innocuous (generically true) assumptions. We now describe his classification scheme, as slightly revised by us in [27].

We assume the system (0) to be fuchsian over $\mathbf{S}$. This condition is precisely defined in 1.2.1; it is essentially equivalent to the existence of fundamental solutions with moderate growth at 0 and $\infty$, as opposed to theta-like growth, like $z^{\log z}$ (see [22]). The idea of Birkhoff is to use local solutions at the two only $q$-invariant points of $\mathbf{S}, 0$ and $\infty$, and to classify fuchsian systems using linear data at 0 and $\infty$ and one matrix connecting a local solution at 0 to one at $\infty$. Here are the main steps. Note the close similitude of steps 1 to 3 with the classical Frobenius-Fuchs method for ordinary differential equations.

1. Any fuchsian system is meromorphically equivalent (near 0 ) to one with constant coefficients. Here, meromorphic equivalence is defined by letting the gauge transformation $F \in G L_{n}(\mathbf{C}(\{z\}))\left(F\right.$ will then automatically belong to $G L_{n}(\mathcal{M}(\mathbf{C}))$ ).

2. Any constant coefficient system can be reduced by linear algebra to 1-dimensional systems: $\sigma_{q} f=c f$ (where $c$ is an exponent) and, if its matrix is not semi-simple, 2-dimensional unipotent systems: $\sigma_{q} g=g+1$. One can build solutions to such elementary systems that are meromorphic on $\mathbf{C}^{*}$ and have moderate growth at 0 and $\infty$, relying on Jacobi's theta function $\Theta_{q}$. We thus obtain the $q$-characters $e_{q, c}$ and the $q$-logarithm $l_{q}$. From this, we get a canonical fundamental solution $e_{q, A}$ for each constant coefficient system $A \in G L_{n}(\mathbf{C})$.

3. Each fuchsian system is therefore endowed with a local solution at $0: X^{(0)}=M^{(0)} e_{q, A^{(0)}}$, where $M^{(0)} \in G L_{n}(\mathcal{M}(\mathbf{C}))$ and $A^{(0)} \in G L_{n}(\mathbf{C})$, and, symmetrically, a local solution at $\infty: X^{(\infty)}=M^{(\infty)} e_{q, A^{(\infty)}}$, the Jordan structures of $A^{(0)}$ and $A^{(\infty)}$ are the the required "linear data".

4. The connection matrix of Birkhoff is then defined to be: $P=\left(X^{(\infty)}\right)^{-1} X^{(0)}$. It has coefficients in the $q$-invariant subfield $\mathcal{M}\left(\mathbf{C}^{*}\right)^{\sigma_{q}}$ of $\mathcal{M}\left(\mathbf{C}^{*}\right)$, that is, in the field of meromorphic functions on the elliptic curve $\mathbf{E}_{q}=\mathbf{C}^{*} / q^{\mathbf{Z}}$.

Now, Birkhoff's theorem says that, given linear data at 0 and at $\infty$ and an invertible elliptic matrix, one can recover a system (0) well defined up to rational equivalence. A precise formulation can be found in [27].

Birkhoff's method seems to lend itself easily to a reformulation in the style of RiemannHilbert's classification scheme, via local systems and representations of the fundamental group. The local linear data should allow one to compute local monodromy transformations around 0 and $\infty$, so that a groupoid with two base points naturally appears. Then these base points should be connected by paths from 0 to $\infty$ : here, the representation has a natural counterpart, the connection matrix. But the latter has elliptic coefficients, so that one does not end up with algebraic matrices over the complex numbers, as in the classical case: the field of "constants" of the theory, $\mathcal{M}\left(\mathbf{C}^{*}\right)^{\sigma_{q}}=\mathcal{M}\left(\mathbf{E}_{q}\right)$, is too big.

\subsection{Adapting Picard-Vessiot theory}

The first break through the difficulties caused by the big constant field $\mathcal{M}\left(\mathbf{E}_{q}\right)$ was made by Etingof in [14]. He adapted Picard-Vessiot theory to the case of regular $q$-difference systems, those such that $A(0)=A(\infty)=I_{n}$. Etingof defines and builds Picard-Vessiot extensions and shows the related $q$-difference Galois group to be generated by the values (at authorized 
points) $P(a)^{-1} P(b) \in G L_{n}(\mathbf{C})$, where $P$ is the connection matrix. However, in the case of nonregular systems, elliptic functions spontaneously arise in yet another way: if $c, d \in \mathbf{C}^{*}$, then, along with the "legal" solution $e_{q, c d}$ to the equation $\sigma_{q} f=c d f$, there is also $e_{q, c} e_{q, d}$, so that $\phi(c, d)=\frac{e_{q, c} e_{q, d}}{e_{q, c d}}$ is elliptic. Any field containing these basic solutions will contain all elliptic functions, and it is easy to prove that there is no way to trivialize the cocycle $\phi(c, d)$ while using "true functions", so that there seems to be no hope for a Picard-Vessiot theory with a group defined over $\mathbf{C}$ even in the fuchsian case. The second breakthrough was accomplished by van der Put and Singer in [20], using symbolic solutions. They build a Picard-Vessiot theory with constant field $\mathbf{C}$. They are then able to solve the problem in total generality, including the case of irregular (nonfuchsian) systems.

However, our work is part of a program that requires a function theoretic attack at these problems. First, there is the link of $q$-difference equations to ordinary differential equations through $q$-analogies. When $q \rightarrow 1, q$-analogs of special functions (like Heine's basic hypergeometric series, see the "bible" [15]) "tend to" their classical counterpart. One may wish to follow Galois groups along such a confluence. Some results in this direction were expounded in [27]. They receive here substantial extensions. This is of obvious interest in mathematical physics, with the present ubiquity of $q$-deformations. Second, the appearance (and central importance) of Jacobi's theta functions, elliptic functions and complex elliptic curves in the landscape unveil rich geometric structures. We build explicit galoisian automorphisms and give them a geometric interpretation, which allows us to exhibit a reasonable candidate for the role of fundamental group, that is, a finitely generated and finitely presented Zariski-dense subgroup of the Galois group. This is in analogy with Schlesinger's theorem (see [4,7]) and with the topological flavour of the classical Riemann-Hilbert correspondence, where fuchsian differential equations are classified by monodromy representations (see [10]) ${ }^{1}$.

As noted before, the use of "true functions" as $q$-characters forces on us a big constant field $\mathcal{M}\left(\mathbf{E}_{q}\right)$. Yet, in our version of the classification theorem of Birkhoff, automorphisms of fuchsian objects are classified by complex matrices. The root of this fact is that we authorize as unique "legal model" for the equation $\sigma_{q} f=c f$ the one function $e_{q, c}$ and nobody else, thereby rigidifying a lot the situation. The corresponding drawback is that we cannot multiply solutions: $e_{q, c} e_{q, d}$ is not legal, only $e_{q, c d}$ is; we do not even have an algebra of solutions. Therefore, to produce a Galois group, we turn to Tannaka duality (see $[12,11]$ ). This has already been used in this context twice: by van der Put and Singer in [20] for one, then by Yves André in his work [1], where deformation results are proved for difference and differential Galois groups. The goal of this paper is therefore to give a tannakian formulation of the classification theorem of Birkhoff, while using as basic objects uniform analytic functions. We now list our main results (they are detailed in Section 0.4).

The local category $\mathcal{E}_{f}^{(0)}$ of $q$-difference systems is naturally equivalent to the $\mathbf{C}$-linear neutral tannakian category $F i b_{p}\left(\mathbf{E}_{q}\right)$ of flat vector bundles over the elliptic curve $\mathbf{E}_{q}$. There is a naturally defined local Galois groupoid $G^{(0)}$ of $\mathcal{E}_{f}^{(0)}$ with base set $\mathbf{C}^{*}$ and we compute it explicitly, as well as the local Galois group, also called $G^{(0)}$. We build explicit elements of the group $G^{(0)}$, and we want to see them as loops ${ }^{2}$; then, we single out two commuting loops with a nice topological

\footnotetext{
${ }^{1}$ To these arguments, one should add that the oldest historical motivation for the $q$-world lies in magic identities by Gauss, Euler, Jacobi, Ramanujan ... (see [21]). These involve classical analytical functions and one may hope for a geometric understanding of them. One must also mention that $q$-difference equations are a possible intermediate step to understand the mysterious analogy between irregular linear ordinary differential equations and wildly ramified phenomena in positive characteristic (see [24]).

${ }^{2}$ By nicknaming "loop" a galoisian automorphism (i.e. a tensor automorphism of a fibre functor), we just go one little step beyond the terminology introduced by Katz in [18], 1.1.2.1.
} 
interpretation as "fundamental loops of an infinitesimal elliptic curve". The group they generate is Zariski-dense in $G^{(0)}$. We see it as the local fundamental group.

The global category $\mathcal{E}_{f}$ of $q$-difference systems is equivalent to the $\mathbf{C}$-linear neutral tannakian category of triples $\left(A^{(0)}, M, A^{(\infty)}\right)$, made up of two flat vector bundles and a meromorphic isomorphism between. Evaluating such isomorphisms at non singular points provides us with galoisian isomorphisms ("paths") in the global Galois groupoid $G$. The local groupoids $G^{(0)}$ and $G^{(\infty)}$ together with these paths generate a Zariski-dense subgroupoid of $G$. For regular abelian objects with prescribed singular locus, the Galois group is Zariski-generated by the values of the connection matrix. Using methods from geometric class field theory, one can classify all regular abelian representations with prescribed singular locus $S$ of the global Galois group as representations of an explicit affine group.

Last, we describe explicitly the confluence of the generators of the global Galois group to elements of a differential Galois group when $q$ tends to 1 .

\subsection{Contents of this paper}

Let us now describe more precisely the organisation of this paper. In Section 1, we review some basic properties of linear $q$-difference systems with rational coefficients. In Section 1.1, we briefly recall general algebraic properties of $q$-difference systems and $q$-difference modules, mostly adapted from [20]. In Section 1.2, we define the category $\mathcal{E}_{f}$ of fuchsian q-difference systems, a neutral tannakian category over $\mathbf{C}$, and we summarize the first part of our previous work [27], about local solutions and classification of such systems.

Sections 2 and 3 contain the core of this paper, the construction and tentative description of the local and global Galois groups and groupoids of the category of fuchsian $q$-difference systems. Section 2 deals with the local setting and Section 3 with the glueing of the local descriptions at 0 and $\infty$. In 2.1 , we study the local category $\mathcal{E}_{f}^{(0)}$; we consider here as localisation at 0 the action to allow for morphisms with coefficients defined locally for the transcendant topology. We find a particularly simple equivalent category $\mathcal{P}^{(0)}$ of local models related with the category $\mathcal{R}$ of complex representations of $\mathbf{Z}$. In Section 2.2, we exploit this link to exhibit a $\mathbf{C}^{*}$-indexed family of fibre functors $\omega_{z_{0}}^{(0)}$ extending the canonical fibre functor $\omega$ on $\mathcal{R}$, allowing us to compute the local Galois groupoid $G^{(0)}$ of $\mathcal{E}_{f}^{(0)}$, with base set $\mathbf{C}^{*}$ from the knowledge of the proalgebraic hull $\mathbf{Z}^{\text {alg }}=\operatorname{Hom}_{\text {grp }}\left(\mathbf{C}^{*}, \mathbf{C}^{*}\right) \times \mathbf{C}$ of $\mathbf{Z}: G^{(0)}$ is thereby identified with a subgroupoid of $\mathbf{Z}^{\text {alg }}=A u t^{\otimes}(\omega)$. We prove:

2.2.2.1. THEOREM (the local Galois groupoid). - With the previous identification of $A u t^{\otimes}(\omega)$ with $\mathbf{Z}^{\text {alg }}$,

$$
I s o^{\otimes}\left(\omega_{z_{0}}^{(0)}, \omega_{z_{1}}^{(0)}\right)=\left\{(\gamma, \lambda) \in \mathbf{Z}^{\text {alg }} \mid \gamma(q) z_{0}=z_{1}\right\} .
$$

The Galois group (also called $G^{(0)}$ ) is then immediately deduced in Corollary 2.2.2.2. Two commuting algebraically independent elements ("loops") $\gamma_{1}$ and $\gamma_{2}$ in the semi-simple component of $G^{(0)}$ are built in 2.2.3. The following density theorem thus provides an analog to the local fundamental group:

2.2.3.5. THEOREM. - The subgroup of $\mathrm{Hom}_{\text {grp }}\left(\mathbf{C}^{*}, \mathbf{C}^{*}\right) \times \mathbf{C}$ whose unipotent component is $\mathbf{Z} \subset \mathbf{C}$ and whose semi-simple component is generated by $\gamma_{1}$ and $\gamma_{2}$ is Zariski-dense in the local Galois group.

According to Weil's correspondence between the degree 0 vector bundles on a compact Riemann surface and the representations of its fundamental group, our category $\mathcal{P}^{(0)}$ of local models is shown to be equivalent to the category of flat vector bundles over the elliptic curve $\mathbf{E}_{q}$ 
in 2.3. Our solutions can be interpreted as sections of these bundles and the singling out of our fundamental solutions is equivalent to a choice of frames.

In Section 3, we start global Galois theory. In 3.1, we define a category $\mathcal{C}$ of connection triples $\left(A^{(0)}, M, A^{(\infty)}\right)$, made up of two local (flat) systems and an isomorphism $M$ between them that is meromorphic over $\mathbf{C}^{*}$. In Proposition 3.1.1.3, we prove that $\mathcal{E}_{f}$ and $\mathcal{C}$ are equivalent tensor categories. The natural projections to the local categories at 0 and $\infty$ equip them with two $\mathbf{C}^{*}$ indexed families of fibre functors $\omega_{z_{0}}^{(0)}$ and $\omega_{z_{0}}^{(\infty)}$. This defines a Galois groupoid $G$ of $\mathcal{C}$ with base set $\mathbf{C}^{*} \amalg \mathbf{C}^{*}$. Evaluating $M$ at a point $z_{0}$ defines a galoisian isomorphism $\Gamma_{z_{0}}$ between the restrictions of $\omega_{z_{0}}^{(0)}$ and $\omega_{z_{0}}^{(\infty)}$ to the tannakian subcategory $\mathcal{C}_{\Sigma}$ of systems with singular locus carried by $\Sigma$; we want to see such an element as a "path". We then get another density result:

3.1.2.3. THEOREM. - The local groupoids at 0 and at $\infty$ (defined and computed in Section 2) together with the paths $\Gamma_{z_{0}}, z_{0} \notin \Sigma$ generate a Zariski-dense subgroupoid of the Galois groupoid of $\mathcal{C}_{\Sigma}$.

In 3.2, we follow more literally Birkhoff and get stuck in many complications due to the bad multiplicative properties of solutions, precisely, the fact that $e_{q, c d} \neq e_{q, c} e_{q, d}$, leading us to a twisted tensor structure and a twisted connection matrix. However, the grubby computations of 3.2 give a more concrete approach and a simple structural description of the global Galois group. Moreover, it is better fitted for the important confluence results of Section 4 . The relation with the point of view of 3.1 is explained in 3.2.3. In both approaches, we have exhibited a lot of "connecting" galoisian isomorphisms (from 0 to $\infty$ ), built from the values of the connection matrix, and we have proven a density lemma; but we want to reduce the uncountable family of generators thus obtained and to make explicit the relations between them. We solve this difficult problem in 3.3 for regular abelian objects. The Galois group is then reduced to its connection component, which, after 3.1.2, is Zariski-generated by the values of the connection matrix. Relative to a prescribed singular locus $S$ we explicitly define and compute in 3.3.2 (Eq. (3), 3.3.2.1 and 3.3.2.2) affine algebraic groups $L_{S, s}, L_{S, s}^{\prime}$ and $L_{S, u}$ and prove:

3.3.2.3. THEOREM. - The abelian regular objects with singularities in $S$ are classified by the representations of the following algebraic group:

$$
\pi_{a b, S, \mathrm{reg}}^{1}=\frac{L_{S, s}}{L_{S, s}^{\prime}} \times L_{S, u} .
$$

In Section 4, we study, along the lines of our previous work [27], the confluence of $q$-difference galoisian automorphisms to differential galoisian automorphisms when $q \rightarrow 1$. This can be seen as an "internal", maybe more explicit, illustration of results by Yves André in [1], relating a family of $q$-difference Galois groups to a differential Galois group.

\section{General facts and conventions}

We fix for the whole paper a complex number $q \in \mathbf{C}$ such that $|q|>1$ and a number $\tau \in \mathcal{H}$ (Poincaré's half plane) such that $q=e^{-2 \imath \pi \tau}$. The only exception is Section 4.1, where $q$ and $\tau$ will be allowed to vary. For any $c \in \mathbf{C}^{*}$, there is a unique pair $(m, d) \in \mathbf{Z} \times \mathbf{C}$ with $c=q^{m} d$, where $d$ belongs to the fundamental annulus: $1 \leqslant|d|<|q|$; we then put $\varepsilon(c)=m$ and $\bar{c}=d$, so that $\varepsilon(c)$ is the integral part of $\frac{\log |c|}{\log |q|}$ and $c=q^{\varepsilon(c)} \bar{c}$.

For any complex regular matrix $A \in G L_{n}(\mathbf{C})$, we write $A=A_{s} A_{u}$ its (multiplicative) Dunford decomposition: $A_{s}$ is semi-simple, $A_{u}$ is unipotent and they commute. Such a decomposition is unique and both factors are polynomials in $A$. Let $f$ be any map: $\mathbf{C}^{*} \rightarrow \mathbf{C}^{*}$. 
Write $A_{s}=Q \operatorname{diag}\left(c_{1}, \ldots, c_{n}\right) Q^{-1}$. Then, the matrix $Q \operatorname{diag}\left(f\left(c_{1}\right), \ldots, f\left(c_{n}\right)\right) Q^{-1}$ depends on $A_{s}$ only and we write it $f\left(A_{s}\right)$. Except otherwise explicitly stated, we shall then write $f(A)=f\left(A_{s}\right)$. One exception to this last convention is that $\bar{A}=\overline{A_{s}} A_{u}$, so that $A=q^{\varepsilon(A)} \bar{A}$. Another exception appears when we define $e_{q, A}$ in 1.2.2. Also, note the following general fact: if $S A=B S$, then, for any map $f: \mathbf{C}^{*} \rightarrow \mathbf{C}^{*}$ and any $\lambda \in \mathbf{C}$, one has $S f\left(A_{s}\right)=f\left(B_{s}\right) S$ and $S A_{u}^{\lambda}=B_{u}^{\lambda} S$; here, of course,

$$
A_{u}^{\lambda}=\sum_{k \geqslant 0}\left(\begin{array}{l}
\lambda \\
k
\end{array}\right)\left(A_{u}-I_{n}\right)^{k}
$$

(actually, a finite sum).

\section{Preliminary results}

\subsection{Difference systems and difference modules}

Most of the general formalism here is expounded in $[20]^{3}$. Let $(K, \sigma)$ be a difference field: $K$ is a field and $\sigma$ is an automorphism of $K$. We shall also, without further notice, denote by $\sigma$ the canonical extensions to the vector spaces of matrices, or of row or column vectors. The $q$-difference equation of order $n$ :

$$
\sigma^{n} f+a_{1} \sigma^{n-1} f+\cdots+a_{n} f=0, \quad a_{1}, \ldots, a_{n} \in K, a_{n} \neq 0
$$

can be put into system form as a $q$-difference system of rank $n$ :

$$
\sigma X=A X, \quad A \in G L_{n}(K) .
$$

Conversely, any such system is equivalent to such an equation via the gauge equivalence defined by: $A \sim(\sigma F)^{-1} A F, F \in G L_{n}(K)$. This is a consequence of Birkhoff's cyclic vector lemma (see [27], Appendix B or [13]). As a consequence, from now on, we won't distinguish between equations and systems.

\subsubsection{The category of difference modules}

The system (2) can in turn be modelled more intrinsically as a difference module $\left(K^{n}, \Phi\right)$ by putting $\Phi: K^{n} \rightarrow K^{n} X \mapsto A^{-1} \sigma X$, where a difference module over the difference field $K$ (more properly, over $(K, \sigma)$ ) is a finite dimensional $K$-vector space $M$ equipped with a $\sigma$-linear automorphism $\Phi_{M}$ (that is, a group automorphism such that $\Phi_{M}(x m)=\sigma(x) \Phi_{M}(m)$ ). Then $\Phi_{M}$ is actually linear over the constant subfield:

$$
C_{K}=K^{\sigma}=\{x \in K \mid \sigma(x)=x\} .
$$

A morphism $f:\left(M, \Phi_{M}\right) \rightarrow\left(N, \Phi_{N}\right)$ is a $K$-linear map such that $\Phi_{N} \circ f=f \circ \Phi_{M}$. We shall usually write $M, f: M \rightarrow N$, etc., the difference module structure being implicit. Also, we write $r(M)$ for the rank of the difference module $M$, that is, its dimension as a $K$-vector space. We thus obtain the category $\operatorname{DiffMod}(K, \sigma)$ of difference modules over the difference field $(K, \sigma)$. According to [20], this is a $C_{K}$-linear rigid abelian tensor category (see [12] and [11]). Clearly,

\footnotetext{
${ }^{3}$ A different formalism is presented in [1], which introduces a notion of non commutative connection.
} 
forgetting the difference structure (i.e. the automorphism $\Phi_{M}$ ) provides us with a fibre functor from $\operatorname{DiffMod}(K, \sigma)$ to the category $\operatorname{Vect}_{K}^{f}$ of finite dimensional $K$-vector spaces, thus making $\operatorname{DiffMod}(K, \sigma)$ a $C_{K}$-linear tannakian category neutralized by $K$ (see [12]).

\subsubsection{The category of difference systems}

Choosing an ordered basis for each finite dimensional vector space over $K$ allows one to replace the category $\operatorname{Vect}_{K}^{f}$ by its essential full subcategory with objects the $K^{n}(n \in \mathbf{N})$; then the morphisms $K^{n} \rightarrow K^{p}$ can be identified with the matrices in $M_{p, n}(K)$. This subcategory is equivalent, as an abelian category, to $V e c t_{K}^{f}$. To have an equivalence qua tensor categories, it is enough to consistently choose an order on the product of any two ordered bases. For instance, choosing the lexicographic order gives bijections:

$$
\left\{\begin{array}{l}
\left\{1, \ldots, n_{1}\right\} \times\left\{1, \ldots, n_{2}\right\} \rightarrow\left\{1, \ldots, n_{1} n_{2}\right\}, \\
\left(i_{1}, i_{2}\right) \mapsto i_{1}+n_{1}\left(i_{2}-1\right) .
\end{array}\right.
$$

We thus obtain well defined isomorphisms:

$$
K^{n_{1}} \underset{K}{\otimes} K^{n_{2}} \rightarrow K^{n_{1} n_{2}} \quad \text { and } \quad M_{p_{1}, n_{1}}(K) \underset{K}{\underset{K}{\otimes}} M_{p_{2}, n_{2}}(K) \rightarrow M_{p_{1} p_{2}, n_{1} n_{2}}(K) .
$$

The resulting tensor category has trivial (i.e. identity) associativity and unity constraints (but it is not so for the commutativity constraint).

In the same spirit, define the category DiffE $q(K, \sigma)$ of difference equations over the difference field $(K, \sigma)$ : it has as objects the pairs $\left(K^{n}, A\right)$ where $n \in \mathbf{N}$ and $A \in G l_{n}(K)$; and, as morphisms from $\left(K^{n}, A\right)$ to $\left(K^{p}, B\right)$, the matrices $F \in M_{p, n}(K)$ such that $(\sigma F) A=B F$ (the composition is the natural one). We shall often simply denote by $A$ the object $\left(K^{n}, A\right)$ and identify it with the difference equation $\sigma X=A X$; the main reason to make the base space $K^{n}$ explicit is to give a more natural notation to the forgetful functor $\left(K^{n}, A\right) \sim K^{n}$. To obtain $\operatorname{DiffE} q(K, \sigma)$ as a tensor model of $\operatorname{DiffMod}(K, \sigma)$, we define the tensor product of two objects by: $\left(K^{n_{1}}, A_{1}\right) \otimes\left(K^{n_{2}}, A_{2}\right)=\left(K^{n_{1} n_{2}}, A_{1} \otimes A_{2}\right)$, with the previous identification of $A_{1} \otimes A_{2}$ to a matrix in $M_{n_{1} n_{2}}(K)$; and the tensor product of two morphisms $F_{i}:\left(K^{n_{i}}, A_{i}\right) \rightarrow\left(K^{p_{i}}, B_{i}\right)$ $(i=1,2)$ as $F_{1} \otimes F_{2}$, similarly identified with a matrix in $M_{p_{1} p_{2}, n_{1} n_{2}}(K)$. From 1.1.1, we draw that the above constructions make DiffE $q(K, \sigma)$ into a rigid $C_{K}$-linear abelian tensor category equivalent to $\operatorname{DiffMod}(K, \sigma)$. It is tannakian and neutralized by $K$. The basic relevant linear and tensor constructions are detailed in [26] and in [29]. In particular, the unit $\underline{1}$ is $(K, 1)$.

\subsubsection{Functors of solutions}

The functor of global sections on $\operatorname{DiffMod}(K, \sigma)$ is the functor $\Gamma=\operatorname{Hom}(\underline{1},-)$. The elements of $\Gamma(M)$ are precisely the fixed vectors of $\Phi_{M}$ in $M$. We clearly get a left exact functor to the category of $C_{K}$-vector spaces. From the inequality $\operatorname{dim}_{C_{K}} \Gamma(M) \leqslant r(M)$ (which follows from the " $q$-analogue of the Wronskian lemma", see [13], I.1.2), follows that this functor actually goes to the category $\operatorname{Vect}_{C_{K}}^{f}$. Now let $\left(K^{\prime}, \sigma^{\prime}\right)$ be an extension of $(K, \sigma)$, that is, $K^{\prime}$ is an extension of $K$ and $\sigma_{\mid K}^{\prime}=\sigma$. The naturally defined base change functor from $\operatorname{Diff} \operatorname{Mod}(K, \sigma)$ to $\operatorname{DiffMod}\left(K^{\prime}, \sigma^{\prime}\right)$ is exact and $\otimes$-preserving. Combining these constructions yields a functor $M \sim\left(M \otimes K^{\prime}\right)^{\sigma^{\prime}}$.

In matrix terms, we associate to a system (2) the $C_{K^{\prime}}$-space $S_{K^{\prime}}(A)$ of solutions in $K^{\prime n}$. We thereby obtain a functor from $\operatorname{DiffE} q(K, \sigma)$ to $\operatorname{Vect}_{C_{K^{\prime}}}^{f}$ defined by:

$$
\left\{\begin{array}{l}
A \sim S_{K^{\prime}}(A), \\
(F: A \rightarrow B) \sim\left(U \mapsto F U: S_{K^{\prime}}(A) \rightarrow S_{K^{\prime}}(B)\right) .
\end{array}\right.
$$


Call fundamental (matrix) solution of the system with matrix $A \in G L_{n}(K)$ over the extension $K^{\prime}$ a matrix solution $X \in G l_{n}\left(K^{\prime}\right)$. The rank of the $C_{K^{\prime}}$ vector space $S_{K^{\prime}}(A)$ is exactly $n$ if and only if there is a fundamental solution. If all systems have a fundamental solution in $K^{\prime}$, then the functor of solutions is a fibre functor. However, in general, functors of solutions are neither right exact, nor faithful, nor $\otimes$-compatible.

\subsection{Fuchsian equations}

\subsubsection{The category $\mathcal{E}_{f}$ of fuchsian equations}

We shall define here the category $\mathcal{E}$ of linear $q$-difference equations with rational coefficients and its subcategory $\mathcal{E}_{f}$ of fuchsian equations. We shall use the following fields of functions: $\mathbf{C}(z)$, the field of rational functions; $\mathcal{M}(\mathbf{C})$, the field of meromorphic functions over $\mathbf{C}$; $\mathcal{M}\left(\mathbf{C}_{\infty}\right)$, the field of meromorphic functions over $\mathbf{C}_{\infty}=\mathbf{S}-\{0\}$; and $\mathcal{M}\left(\mathbf{C}^{*}\right)$, the field of meromorphic functions over $\mathbf{C}^{*}$. Each of these function fields, endowed with the automorphism $\sigma_{q}: f(z) \mapsto f(q z)$, is a difference field. To any of them, we can specialize the preceding constructions.

We are particularly interested in the category of (linear) rational $q$-difference equations, obtained by taking $K=\mathbf{C}(z)$ and $\sigma=\sigma_{q}$. We shall call it $\mathcal{E}=\operatorname{DiffE} q\left(\mathbf{C}(z), \sigma_{q}\right)$. Since the constant field is, in this case, $C_{K}=\mathbf{C}(z)^{\sigma_{q}}=\mathbf{C}, \mathcal{E}$ is a $\mathbf{C}$-linear tannakian category neutralised by $\mathbf{C}(z)$.

We shall say that a system with matrix $A \in G l_{n}(\mathbf{C}(z))$ is strictly fuchsian at 0 if $A(0) \in$ $G l_{n}(\mathbf{C})$. We shall then call fuchsian at 0 a system that is meromorphically (that is, through a gauge transformation with coefficients in $\mathcal{M}(\mathbf{C})$ ) equivalent to a strictly fuchsian one. It was proved in [27], Annexe B, that this definition is equivalent to the classical one (using the Newton polygon). Considering $A(\infty)$ and gauge transformations with coefficients in $\mathcal{M}\left(\mathbf{C}_{\infty}\right)$, we similarly define systems fuchsian (resp. strictly fuchsian) at $\infty$. It was also proved in loc. cit. that an equation fuchsian at 0 and at the same time fuchsian at $\infty$ is rationally (that is, through a gauge transformation with coefficients in $\mathbf{C}(z)$ ) equivalent to one that is strictly fuchsian over $\mathbf{S}$ (i.e. at 0 and $\infty$ ). Such equations we call fuchsian over $\mathbf{S}$, or merely fuchsian. They form a strictly full subcategory $\mathcal{E}_{f}$ of $\mathcal{E}$.

\subsubsection{THEOREM. - The category $\mathcal{E}_{f}$ is a tannakian subcategory of $\mathcal{E}$ over $\mathbf{C}$.}

To see that $\mathcal{E}_{f}$ is closed under tensor operations (including unit, dual and internal Hom) it is plainly enough to consider the case of strictly fuchsian objects, and then it is obvious. Now, from the lemma herebelow, it follows that the kernel in $\mathcal{E}$ of any morphism between fuchsian objects is itself fuchsian. Therefore, it is a kernel in $\mathcal{E}_{f}$. Since duality in the tannakian category $\mathcal{E}$ exchanges kernels with cokernels (this follows from [12, p. 112]), we conclude that $\mathcal{E}_{f}$ is indeed an abelian subcategory of $\mathcal{E}$.

\subsubsection{LEMMA. - In $\mathcal{E}$, any subobject of an object that is fuchsian at 0 is so.}

This is an immediate consequence of the properties of the Newton polygon studied in $[28,29,32]$. For a more explicit analytic proof see [26] (see also [19]).

\subsubsection{Local reduction and local solutions}

We recall, here and in the following section, some results from [27] and [26]. First, define Jacobi's theta function:

$$
\Theta_{q}(z)=\sum_{n \in \mathbf{Z}}(-1)^{n} q^{-n(n-1) / 2} z^{n}
$$


It is holomorphic over $\mathbf{C}^{*}$ with simple zeros on $q^{\mathbf{Z}}$. It satisfies the $q$-difference equation $\Theta_{q}(q z)=-q z \Theta_{q}(z)$. It will be our main brick to build everything. First, one defines the q-logarithm:

$$
l_{q}(z)=z \frac{\Theta_{q}^{\prime}(z)}{\Theta_{q}(z)},
$$

which is meromorphic over $\mathbf{C}^{*}$ with simple poles on $q^{\mathbf{Z}}$ and satisfies the $q$-difference equation $l_{q}(q z)=l_{q}(z)+1$. Then, for each $c \in \mathbf{C}^{*}$, one defines the $q$-character with exponent $c$. First, if $c$ lies in the fundamental annulus $\{z \in \mathbf{C}|1 \leqslant| z|<| q \mid\}$, one puts:

$$
e_{q, c}(z)=\frac{\Theta_{q}(z)}{\Theta_{q}\left(c^{-1} z\right)} .
$$

For $c$ arbitrary, one writes $c=q^{\varepsilon(c)} \bar{c}$, where $\varepsilon(c) \in \mathbf{Z}$ and $\bar{c}$ belongs to the fundamental annulus, and one puts ${ }^{4}$ :

$$
e_{q, c}=z^{\varepsilon(c)} e_{q, \bar{c}} .
$$

Then $e_{q, q^{n}}=z^{n}$ (if $n \in \mathbf{Z}$ ) and each non trivial $e_{q, c}$ is meromorphic over $\mathbf{C}^{*}$ with simple zeros on $q^{\mathbf{Z}}$ and simple poles on $c q^{\mathbf{Z}}$. It satisfies the $q$-difference equation $e_{q, c}(q z)=c e_{q, c}(z)$, as well as various relations as a family: for instance, $e_{q, q c}(z)=z e_{q, c}(z)$, etc.

Now, let $A \in G L_{n}(\mathbf{C})$ with Dunford decomposition $A=A_{s} A_{u}$. If

$$
A_{s}=Q \operatorname{diag}\left(c_{1}, \ldots, c_{n}\right) Q^{-1},
$$

it makes sense to define:

$$
e_{q, A_{s}}=Q \operatorname{diag}\left(e_{q, c_{1}}, \ldots, e_{q, c_{n}}\right) Q^{-1}
$$

Similarly, defining:

$$
e_{q, A_{u}}=A_{u}^{l_{q}}=\sum_{k \geqslant 0}\left(\begin{array}{c}
l_{q} \\
k
\end{array}\right)\left(A_{u}-I_{n}\right)^{k}
$$

makes sense, since $A_{u}$ is unipotent. One then has

$$
\sigma_{q}\left(e_{q, A_{s}}\right)=A_{s} e_{q, A_{s}} \quad \text { and } \quad \sigma_{q}\left(e_{q, A_{u}}\right)=A_{u} e_{q, A_{u}}
$$

and defining

$$
e_{q, A}=e_{q, A_{s}} e_{q, A_{u}}
$$

one gets the canonical fundamental solution of the constant coefficients system $A$. The above equality, besides, is a Dunford decomposition. From the relation $e_{q, q c}(z)=z e_{q, c}(z)$ stems the equality $e_{q, A}=z^{\varepsilon(A)} e_{q, \bar{A}}$.

We shall build solutions with coefficients in the field:

$$
\mathbf{K}_{\mathbf{0}}=\mathcal{M}(\mathbf{C})\left(l_{q},\left(e_{q, c}\right)_{c \in \mathbf{C}^{*}}\right) .
$$

\footnotetext{
${ }^{4}$ This is an innocuous modification with respect to [27].
} 
As noted in the introduction, the constant subfield $\mathbf{K}_{\mathbf{0}}{ }^{\sigma_{q}}$ is precisely equal to $\mathcal{M}\left(\mathbf{C}^{*}\right)^{\sigma_{q}}$, the field $\mathcal{M}\left(\mathbf{E}_{q}\right)$ of elliptic functions: indeed, one inclusion is obvious and the other comes from the classical fact that the family of the cocycle values:

$$
\phi(c, d)=\frac{e_{q, c} e_{q, d}}{e_{q, c d}}=\frac{\Theta_{q}(z) \Theta_{q}\left(c^{-1} d^{-1} z\right)}{\Theta_{q}\left(c^{-1} z\right) \Theta_{q}\left(d^{-1} z\right)}
$$

generates the group $\mathcal{M}\left(\mathbf{E}_{q}\right)^{*}$. Extending the cocycle $\phi$ to semi-simple matrices, one gets a cocycle $\Phi$ of invertible elliptic matrices such that, for any two semi-simple matrices $C, C^{\prime}$, one has $e_{q, C} \otimes e_{q, C^{\prime}}=e_{q, C \otimes C^{\prime}} \Phi\left(C, C^{\prime}\right)$. On the other hand, it is clear that, for unipotent matrices $U, U^{\prime}$, one has $e_{q, U} \otimes e_{q, U^{\prime}}=e_{q, U \otimes U^{\prime}}$. Thus, for any two invertible matrices $A, A^{\prime}, \Phi\left(A_{s}, A_{s}^{\prime}\right)$ is exactly the defect of $\otimes$-compatibility of the formation of our canonical solutions $e_{q,-}$ :

$$
e_{q, A} \otimes e_{q, A^{\prime}}=e_{q, A \otimes A^{\prime}} \Phi\left(A_{s}, A_{s}^{\prime}\right) .
$$

To build explicit solutions, we follow closely the classical way for differential equations: see $[17,36]$. We consider local reduction at 0 , the case of $\infty$ being similar.

First, any fuchsian system reduces by definition to a strictly fuchsian one through a rational gauge transformation. Any strictly fuchsian system reduces similarly to a nonresonant one, that is, such that no two distinct exponents (eigenvalues of $A(0)$ ) are congruent modulo $q^{\mathbf{Z}}$. This process involves some non canonical choices.

Second, any non resonant system $A$ is equivalent to the constant coefficients system $A(0)$. This is obtained by solving the functional equation with initial condition:

$$
\left\{\begin{array}{l}
F(0)=I_{n}, \\
\left(\sigma_{q} F\right) A(0)=A F
\end{array}\right.
$$

with $F$ a convergent power series. The equivalent equation $\sigma_{q} F=A F(A(0))^{-1}$ then entails that $F$ is actually meromorphic over $\mathbf{C}$ (the polarity will be precised below). The transformation matrix $F$ is unique.

It follows that any system $A$ that is fuchsian at 0 has a fundamental solution

$$
X^{(0)}=M^{(0)} e_{q, A^{(0)}}
$$

where $M^{(0)} \in G L_{n}(\mathcal{M}(\mathbf{C}))$ and $A^{(0)} \in G L_{n}(\mathbf{C})$; the latter can be assumed to be non resonant and have all its eigenvalues in the fundamental annulus. One can also add more rigid normalising conditions, like sorted eigenvalues (with respect to an arbitrary order on the fundamental annulus) and sorted sizes of the Jordan blocks (see [27]). Defining the singular locus of a matrix $M$ to be:

$$
\mathcal{S}(M)=\{\text { poles of } M\} \cup\left\{\text { poles of } M^{-1}\right\}=\{\text { poles of } M\} \cup\{\text { zeros of } \operatorname{det} M\},
$$

one finds the singular locus of the meromorphic part:

$$
\mathcal{S}\left(M^{(0)}\right)=q^{-\mathbf{N}^{*}} \mathcal{S}(A)
$$

The singular locus of the "log-car" part $e_{q, A^{(0)}}$ is easily deduced from its definition. 


\subsubsection{Connection matrix and global classification}

We now assume $A$ to be fuchsian over $\mathbf{S}$ and attach to it local solutions $X^{(0)}$ and $X^{(\infty)}$ as defined in 1.2.2. We then define Birkhoff's connection matrix to be:

$$
P=\left(X^{(\infty)}\right)^{-1} X^{(0)}
$$

It is clearly an elliptic invertible matrix. We attach to the system $A$ the (non uniquely defined) triple $\left(A^{(0)}, P, A^{(\infty)}\right)$ (using the notations of 1.2.2). Changing the non unique choices, or changing $A$ to a rationally equivalent system $B$ may produce another triple $\left(B^{(0)}, Q, B^{(\infty)}\right)$. If, in addition, one assumes normalised log-car parts (as described above), one must have $\left(A^{(0)}, A^{(\infty)}\right)=\left(B^{(0)}, B^{(\infty)}\right)$ and there exist constant invertible matrices $R, S$ such that $R Q=P S$ plus another commutation condition more precisely explained in [27]. There is then a bijective correspondence between classes of fuchsian systems modulo rational equivalence and classes of triples. The surjectivity follows from Birkhoff's lemma, originating in [5], a modern version of which can be found in [2]. The way we use it is detailed in [27], 2.2 and 2.3. The tannakian counterpart of this bijection will be the content of 3.1 and 3.2.

\subsubsection{Functors of solutions}

Specializing the constructions of 1.1.3 to the extension $K^{\prime}=\mathcal{M}\left(\mathbf{C}^{*}\right)$ of $K=\mathbf{C}(z)$ provides us with a functor of solutions $\omega^{*}=S_{K^{\prime}}$ from $\mathcal{E}$ to $\operatorname{Vect}{ }_{\mathcal{M}\left(\mathbf{E}_{q}\right)}^{f}$. In order to make $\omega^{*}$ a fibre functor, it would be enough to guarantee, for each equation $A$, the existence of a fundamental matrix solution $X \in G l_{n}\left(\mathcal{M}\left(\mathbf{C}^{*}\right)\right)$. It follows from 1.2.3 that, for $A$ in $\mathcal{E}_{f}$, there is indeed such a fundamental matrix solution and the rank of $\omega^{*}(A)$ is equal to the order of $A$. Thus, the restriction of $\omega^{*}$ to $\mathcal{E}_{f}$ is a fibre functor on $\mathcal{E}_{f}$ over the field $\mathcal{M}\left(\mathbf{E}_{q}\right)$. However, we are rather looking for a fibre functor on $\mathcal{E}_{f}$ over the field $\mathbf{C}$.

We shall nearly build such functors from the local solutions at 0 and $\infty: X^{(0)}=M^{(0)} e_{q, A(0)}$ and $X^{(\infty)}=M^{(\infty)} e_{q, A(\infty)}$. These solutions are not canonical, but, by choosing such a pair for every equation, one is led to the definition of two vector spaces of finite rank over the complex numbers. We thus define the functors of solutions at 0 and $\infty$ on $\mathcal{E}_{f}$ with values in $\operatorname{Vect}_{\mathbf{C}}^{f}$ as:

$$
\omega^{(0)}:\left\{\begin{array}{l}
A \sim X^{(0)} \mathbf{C}^{n}, \\
(F: A \rightarrow B) \sim(U \mapsto F U)
\end{array}, \quad \omega^{(\infty)}:\left\{\begin{array}{l}
A \sim X^{(\infty)} \mathbf{C}^{n}, \\
(F: A \rightarrow B) \sim(U \mapsto F U) .
\end{array}\right.\right.
$$

Let $F: A_{1} \rightarrow A_{2}$ be a morphism in $\mathcal{E}_{f}$. Then, with obvious notations, $F X_{1}^{(0)}$ is a solution of $A_{2}$, hence of the form $X_{2}^{(0)} S^{(0)}$ where $S^{(0)}$ is elliptic; similarly at $\infty$. That the above definition makes sense comes from the fact that $S^{(0)}$ and $S^{(\infty)}$ actually have coefficients in C. This follows from the following lemma, which we formulate in a slightly more general guise for further use.

1.2.4.1. LemMA. - Let $A, A^{\prime}$ have constant coefficients and $F, M, M^{\prime}$ have coefficients in $\mathcal{M}(\mathbf{C})$ and suppose that:

$$
F\left(M e_{q, A}\right)=\left(M^{\prime} e_{q, A^{\prime}}\right) S
$$

where $S$ is elliptic. Then $S$ has constant coefficients.

From the conjugacy properties of matrices $e_{q, X}$ (viz, $e_{q, Q X Q^{-1}}=Q e_{q, X} Q^{-1}$ ), one sees that $A$ and $B$ can be assumed to be upper triangular. We write $A=A_{s} A_{u}, A^{\prime}=A_{s}^{\prime} A_{u}^{\prime}$ their Dunford decompositions, so that $e_{q, A}=e_{q, A_{s}} e_{q, A_{u}}$ and $e_{q, A^{\prime}}=e_{q, A_{s}^{\prime}} e_{q, A_{u}^{\prime}}$. From the formulae $e_{q, c}=z^{\varepsilon(c)} e_{q, \bar{c}}$, we see that we can assume all exponents (eigenvalues) of $A, A^{\prime}$ to lie in the 
fundamental annulus $1 \leqslant|c|<|q|$. We then write:

$$
\left(M^{\prime} e_{q, A_{u}^{\prime}}\right)^{-1} F\left(M e_{q, A_{u}}\right)=e_{q, A_{s}^{\prime}} S\left(e_{q, A_{s}}\right)^{-1} .
$$

The $(i, j)$ coefficient of the right hand side is $s_{i, j} \frac{e_{q, c_{i}^{\prime}}}{e_{q, c_{j}}}$, a chararacter (since $s_{i, j}$ is elliptic) with coefficients in $\mathcal{M}(\mathbf{C})\left[l_{q}\right]$, because of the left hand side. It follows from the independence properties of $q$-characters ([27], appendix) and from the location of $c_{j}^{\prime}, c_{i}$ in the fundamental annulus, that it must be trivial: $s_{i, j}$ must be a constant.

Note that $s_{i, j}$ must be null if $c_{j}^{\prime} \neq c_{i}$, which amounts to say that $A_{s}^{\prime} S=S A_{s}$. This being true after reduction to the fundamental annulus, the more general equality is:

$$
\overline{A_{s}^{\prime}} S=S \overline{A_{s}} .
$$

We then have

$$
\left(M^{\prime-1} F M\right) e_{q, A_{u}}=e_{q, A_{u}^{\prime}} S
$$

expanding over $\mathcal{M}(\mathbf{C})\left[l_{q}\right]$ and identifying corresponding powers of $l_{q}$ (which is transcendental over $\mathcal{M}(\mathbf{C})$, see loc. cit.) entails

$$
\left(M^{\prime-1} F M\right) A_{u}=A_{u}^{\prime} S
$$

These formulae are not affected by the conjugacies we used at the beginning, but do depend on the reduction to the fundamental annulus.

On the other hand, the functors $\omega^{(0)}, \omega^{(\infty)}$ are not $\otimes$-compatible. In Section 2, we shall eventually build more easily fibre functors for fuchsian equations while avoiding the choice of particular solutions.

\subsubsection{Singularities and exponents}

In order to compute the "connection component" of the Galois groupoid in Section 3, we shall need to evaluate the connection matrix $P$ or its meromorphic component $M$ at various points of $\mathbf{C}^{*}$, avoiding their singularities. Since $P$ is elliptic and $M$ satisfies a simple $q$ difference equation, these singularities are defined modulo $q^{\mathbf{Z}}$ and may actually be seen as points in $\mathbf{E}_{q}$. They either come from the equation $A$ at stake, or from its local linear data at 0 and $\infty$. Let $\left(A^{(0)}, P, A^{(\infty)}\right)$ be a triple coming from an object $A$ of $\mathcal{E}_{f}$ and write, as usual, $X^{(0)}=M^{(0)} e_{q, A^{(0)}}$ and $X^{(\infty)}=M^{(\infty)} e_{q, A^{(\infty)}}$ the local solutions used to compute $P$. Then, the singularities of $P$ respectively come from:

1. $\mathcal{S}(A)$ for the meromorphic part $M=\left(M^{(\infty)}\right)^{-1} M^{(0)}$. Precisely, $\mathcal{S}(M) \subset q^{\mathbf{z}} \mathcal{S}(A)$.

2. $S p(A(0)), S p(A(\infty))$ for the semi-simple components of the $e_{q, A(-)}$ parts. Since reduction to a constant matrix is not unique, these exponents of $A$ are actually defined up to a factor in $q^{\mathbf{Z}}$.

3. $l_{q}$, the $q$-logarithm, in case $A(0)$ or $A(\infty)$ is not semi-simple.

On the other hand, we want to define $\otimes$-stable categories only, so that we have to allow for multiplication of the exponents (and the inverse, to have stability under dualisation). We thus define two categories $\mathcal{E}_{f, \Sigma}$ and $\mathcal{E}_{f, \Sigma, C}$. Let $\Sigma$ be a finite subset of $\mathbf{C}^{*}$. Then $\mathcal{E}_{f, \Sigma}$ is the full subcategory of $\mathcal{E}_{f}$ whose objects have all singularities in $q^{\mathbf{Z}_{\Sigma}}$. Let moreover $C$ be a finitely generated subgroup of the group $\mathbf{C}^{*}$. Then $\mathcal{E}_{f, \Sigma, C}$ is the full subcategory of $\mathcal{E}_{f, \Sigma}$ whose objects have all exponents in $q^{\mathbf{z}} C$. They are obviously (strictly full) tannakian subcategories of $\mathcal{E}_{f}$ which is their inductive limit. More precisely, the tannakian subcategory $\langle A\rangle$ generated by an arbitrary 
object $A$ (see [12]) is contained in such a $\mathcal{E}_{f, \Sigma, C}$. We observe that, for $A$ in $\mathcal{E}_{f, \Sigma}, \mathcal{S}(M) \subset q^{\mathbf{Z}}$; and for $A$ in $\mathcal{E}_{f, \Sigma, C}, \mathcal{S}(P) \subset q^{\mathbf{Z}}(\Sigma \cup C)$. Note that we impose (yet) no control on the $q$-logarithm and unipotent parts. This will be done further below. Also note that we do not control separately the exponents at 0 and $\infty$, since we mainly seek to avoid the singularities of $P$. $q^{\mathbf{z}} \Sigma$

1.2.5.1. Proposition. $-A$ is in $\mathcal{E}_{f, \Sigma, C}$ if and only if its exponents are in $C$ and $\mathcal{S}(M) \subset$

This follows from a precise use of Birkhoff's lemma (see [27], 2.3.1). Here, of course, $M=e_{q, A^{(\infty)}} P\left(e_{q, A^{(0)}}\right)^{-1}$.

We now consider regular equations at 0 , that is, such that $A(0)=I_{n}$. For such an equation, the product formula:

$$
\prod_{i \geqslant 1} A\left(q^{-i} z\right) \stackrel{\text { def }}{=} A\left(q^{-1} z\right) A\left(q^{-2} z\right) \cdots
$$

defines a solution holomorphic at 0 and meromorphic on $\mathbf{C}$. Thus, if $A$ is regular at 0 and at $\infty$, we obtain an explicit formula for the connection matrix $P$ (rather similar to Jacobi's triple product formula for the function $\theta$ ), showing it to have as a singular locus exactly $q^{\mathbf{z}} \mathcal{S}(A)$. In this case, $M=P$. Now, call more generally regular (at 0 ) an equation that is equivalent to an $A$ such that $A(0)=I_{n}$. We define the category $\mathcal{E}_{f \text {,reg }}$ as the strictly full subcategory of $\mathcal{E}_{f}$ whose objects are equations regular at 0 and $\infty$. It is clearly a tannakian subcategory of $\mathcal{E}_{f}$, the one studied by Etingof in [14].

\section{Local theory}

The usual method in differential Galois theory for building fibre functors is to use spaces of solutions. But our solutions have bad multiplicative properties: any choice of solutions uniform over $\mathbf{C}^{*}$ will lead to some complicated twisting of the tensor product and of the connection matrix. We thus avoid to single out special fundamental solutions; in essence, this amounts to use sections of vector bundles without expressing them in frames.

\subsection{Localisation at 0 and at $\infty$}

\subsubsection{The categories $\mathcal{E}_{f}^{(0)}$ and $\mathcal{E}_{f}^{(\infty)}$}

The category $\mathcal{E}_{f}^{(0)}$ has the same objects as $\mathcal{E}_{f}$, but morphisms from $A$ (of rank $n$ ) to $B$ (of rank $p$ ) are all $F \in M_{p, n}(\mathbf{C}(\{z\}))$ such that $\left(\sigma_{q} F\right) A=B F$. The functional equation $\sigma_{q} F=B F A^{-1}$ entails that the coefficients of $F$ actually belong to $\mathcal{M}(\mathbf{C})$.

We define similarly $\mathcal{E}_{f}^{(\infty)}$ (morphisms with coefficients in $\mathcal{M}\left(\mathbf{C}_{\infty}\right)$ ) and $\mathcal{E}_{f}^{(*)}$ (morphisms with coefficients in $\mathcal{M}\left(\mathbf{C}^{*}\right)$ ). These are plainly abelian rigid tensor categories, but $\mathcal{E}_{f}^{(*)}$ is $\mathcal{M}\left(\mathbf{E}_{q}\right)$ linear while $\mathcal{E}_{f}^{(0)}$ and $\mathcal{E}_{f}^{(\infty)}$ are only $\mathbf{C}$-linear (since a solution of $\sigma_{q} f=f$ meromorphic at 0 or at $\infty$ has to be a constant). It is clear that the natural embeddings $\mathcal{E}_{f} \rightarrow \mathcal{E}_{f}^{(0)}, \mathcal{E}_{f} \rightarrow \mathcal{E}_{f}^{(\infty)}$, $\mathcal{E}_{f}^{(0)} \rightarrow \mathcal{E}_{f}^{(*)}$ and $\mathcal{E}_{f}^{(\infty)} \rightarrow \mathcal{E}_{f}^{(*)}$ are $\mathbf{C}$-linear exact faithful $\otimes$-functors. We intend to use this fact in the following way: any fibre functor $\omega$ on $\mathcal{E}_{f}^{(0)}$ will restrict to a fibre functor $\omega^{\prime}$ on $\mathcal{E}_{f}$. This realizes $\operatorname{Gal}\left(\mathcal{E}_{f}^{(0)}\right)=A u t^{\otimes}\left(\omega^{\prime}\right)$ as this subgroup of $\operatorname{Gal}\left(\mathcal{E}_{f}\right)=A u t^{\otimes}(\omega)$ made up of elements natural with respect to all morphisms in $\mathcal{E}_{f}^{(0)}$ (and not only in $\mathcal{E}_{f}$ ). 


\subsubsection{The categories $\mathcal{P}^{(0)}$ and $\mathcal{P}^{(\infty)}$}

We call flat an object of $\mathcal{E}_{f}^{(0)}$ (resp. $\mathcal{E}_{f}^{(\infty)}, \mathcal{E}_{f}^{(*)}$ ) if it is a matrix with coefficients in $\mathbf{C}$. These will be identified with flat vector bundles on $\mathbf{E}_{q}$ in 2.3 . The category $\mathcal{P}^{(0)}\left(\right.$ resp. $\left.\mathcal{P}^{(\infty)}, \mathcal{P}^{(*)}\right)$ is the full subcategory of $\mathcal{E}_{f}^{(0)}$ (resp. $\mathcal{E}_{f}^{(\infty)}, \mathcal{E}_{f}^{(*)}$ ) whose objects are flat objects. These subcategories are obviously $\mathbf{C}$-linear and stable under tensor operations.

2.1.2.1. LEMMA. - They are essential subcategories, that is, the inclusion functors

$$
\mathcal{P}^{(0)} \leadsto \mathcal{E}_{f}^{(0)}, \quad \mathcal{P}^{(\infty)} \leadsto \mathcal{E}_{f}^{(\infty)} \quad \text { and } \quad \mathcal{P}^{(*)} \leadsto \mathcal{E}_{f}^{(*)}
$$

are essentially surjective.

Indeed, this is the content of the reduction to a constant coefficient system by a meromorphic gauge transformation (see 1.2.2).

The following is an immediate consequence:

2.1.2.2. Proposition. $-\mathcal{P}^{(0)}$ is a neutral tannakian category over $\mathbf{C}$, equivalent to $\mathcal{E}_{f}^{(0)}$. Similar statements hold for $\mathcal{P}^{(\infty)}, \mathcal{P}^{(*)}$. As a corollary, $\mathcal{P}^{(0)}$ and $\mathcal{P}^{(\infty)}$ have the same Galois group as $\mathcal{E}_{f}^{(0)}$ and $\mathcal{E}_{f}^{(\infty)}$ respectively.

\subsubsection{Flat objects and equivariant morphisms of representations of $\mathrm{Z}$}

We do everything at 0 , the case of $\infty$ being similar. We call $\mathcal{R}$ the category $\operatorname{Rep}_{\mathbf{C}}(\mathbf{Z})$ of finite dimensional complex representations of $\mathbf{Z}$. These can be considered as pairs $\left(\mathbf{C}^{n}, A\right)(A$ a regular complex matrix of rank $n)$, and the morphisms $F:\left(\mathbf{C}^{n}, A\right) \rightarrow\left(\mathbf{C}^{p}, B\right)$ as matrices $F \in M_{p, n}(\mathbf{C})$ such that $F A=B F$. The category $\mathcal{R}$ is a $\mathbf{C}$-linear neutral tannakian category with the forgetful functor $\omega$ as fibre functor and its Galois group:

$$
\mathbf{Z}^{a l g}=A u t^{\otimes}(\omega)
$$

is the proalgebraic hull of $\mathbf{Z}$. Its structure and the way it operates are recalled in 2.2.1.

2.1.3.1. Proposition. - The natural embedding $\mathcal{R} \rightarrow \mathcal{P}^{(0)}$ is a faithful (but not full), essentially surjective exact $\mathbf{C}$-linear $\otimes$-functor.

The categories at stake have the same objects but $\mathcal{R}$ has only constant morphisms, while $\mathcal{P}^{(0)}$ has all equivariant morphisms (the word will be justified in 2.3). The proof is therefore immediate.

2.1.3.2. LemMA. - Any morphism $F: A \rightarrow B$ in $\mathcal{P}^{(0)}$ is a Laurent polynomial:

$$
F=\sum F_{k} z^{k}
$$

a finite sum where each $F_{k}$ has coefficients in $\mathbf{C}$.

Indeed, one has $\sigma_{q} F=B F A^{-1}$, so that $F$ has only a pole at 0 : otherwise, these would be propagated along a $q$-spiral and would accumulate at 0 , contradicting the meromorphy of $F$. Now, $F$ has a Laurent series $F=\sum F_{k} z^{k}$. Identifying the $z^{k}$ components of the two sides of the functional equation, we get: $q^{k} F_{k} A=B F_{k}$. This is possible with $F_{k} \neq 0$ only if $S p\left(q^{k} A\right) \cap S p(B) \neq \emptyset$ (see, for instance, the lemma in 1.1.3 of [27], also proven in [36] or [37]). Hence the finiteness of the sum. 
2.1.3.3. Proposition. - $\left(\mathbf{C}^{n}, A\right) \leadsto \mathbf{C}^{n}, F \leadsto F\left(z_{0}\right)$ gives a fibre functor $\omega_{z_{0}}^{(0)}$ on $\mathcal{P}^{(0)}$ for any $z_{0} \in \mathbf{C}^{*}$.

The only nontrivial point is the faithfulness. Suppose $F\left(z_{0}\right)=0$. Then the functional equation implies $\forall k \in \mathbf{Z}, F\left(q^{k} z_{0}\right)=0$, thus $F=0$ ( $F$ being a Laurent polynomial).

\subsubsection{COROLLARY. - The local Galois group $G^{(0)}$ is a closed subgroup of $\mathbf{Z}^{\text {alg }}$.}

Since the previous fibre functor restricts to the forgetful functor on $\mathcal{R}$, it follows from [12], Proposition 2.21 that we have realized $G^{(0)}$ as a subgroup of the Galois group $\mathbf{Z}^{\text {alg }}$ of $\mathcal{R}$. Precisely, restriction of the elements of $A u t^{\otimes}\left(\omega_{z_{0}}^{(0)}\right)$ to $\mathcal{R}$ gives a morphism of groups $G^{(0)} \rightarrow$ $\mathbf{Z}^{\text {alg }}$ and, according to loc. cit., this is a closed immersion of proalgebraic groups.

\subsection{The local Galois groupoid and the local monodromy}

\subsubsection{The proalgebraic hull of $Z$}

We summarize here the main results we shall need. More details and complete proofs can be found in [26] and in [31].

Our basic tool for building a fundamental group with some topological meaning is the "hole": $\mathbf{Z}=\pi_{1}\left(\mathbf{C}^{*}\right)$. Its proalgebraic hull $\mathbf{Z}^{\text {alg }}$ (see the beginning of 2.1.3) is commutative. It is the product $\mathbf{Z}_{s}^{\text {alg }} \times \mathbf{Z}_{u}^{\text {alg }}$ of its semi-simple part:

$$
\mathbf{Z}_{s}^{a l g}=H_{o m}{ }_{g r p}\left(\mathbf{C}^{*}, \mathbf{C}^{*}\right),
$$

the group of characters of the abstract group $\mathbf{C}^{*}$, and of its unipotent part:

$$
\mathbf{Z}_{u}^{a l g}=\mathbf{C} .
$$

The fundamental loop in $\pi_{1}\left(\mathbf{C}^{*}\right)$ is represented by $1 \in \mathbf{Z}$; it is a Zariski-generator of $\mathbf{Z}^{\text {alg }}$, where it splits into a semi-simple component: $I d_{\mathbf{C}^{*}} \in \mathbf{Z}_{s}^{\text {alg }}$ and a unipotent component $1 \in \mathbf{Z}_{u}^{\text {alg }}$. We talk of fundamental semi-simple loop and fundamental unipotent loop.

A representation of $\mathbf{Z}$ is the same thing as a pair $(V, f)$ of a finite dimensional complex vector space $V$ and an automorphism $f \in G L(V)$. So let $\left(\mathbf{C}^{n}, A\right), A \in G L_{n}(\mathbf{C})$, be a (standard model of a) generic object of $\mathcal{R}$. Let $A=A_{s} A_{u}$ be the Dunford decomposition (see the end of the introduction). Let $(\gamma, \lambda) \in H_{o m}$ grp $\left(\mathbf{C}^{*}, \mathbf{C}^{*}\right) \times \mathbf{C}$ be a Galoisian automorphism. Then we put:

$$
A^{(\gamma, \lambda)}=\gamma\left(A_{s}\right) A_{u}^{\lambda}=A_{u}^{\lambda} \gamma\left(A_{s}\right)
$$

Here, $\gamma$ acts on $A_{s}$ through its eigenvalues: if $A_{s}=Q \operatorname{diag}\left(c_{1}, \ldots, c_{n}\right) Q^{-1}$, one has

$$
\gamma\left(A_{s}\right)=Q \operatorname{diag}\left(\gamma\left(c_{1}\right), \ldots, \gamma\left(c_{n}\right)\right) Q^{-1} .
$$

Since $A^{(\gamma, \lambda)} \in G L_{n}(\mathbf{C})$, it defines an automorphism of $\mathbf{C}^{n}=\omega\left(\mathbf{C}^{n}, A\right)$. This is precisely how $(\gamma, \lambda)$ is incarnated as an element of $A u t^{\otimes}(\omega)$.

\subsubsection{The local Galois groupoid}

Any fibre functor $\omega_{z_{0}}^{(0)}$ (as defined in 2.1.3.3) obviously restricts to the forgetful functor $\omega$ on $\mathcal{R}$. Therefore, $A u t^{\otimes}\left(\omega_{z_{0}}^{(0)}\right)$ is a subgroup (and $I s o^{\otimes}\left(\omega_{z_{0}}^{(0)}, \omega_{z_{1}}^{(0)}\right)$ a subset) of $A u t^{\otimes}(\omega)$.

2.2.2.1. THEOREM (the local Galois groupoid). - With the previous identification of $A u t^{\otimes}(\omega)$ with $\mathbf{Z}^{\text {alg }}$,

$$
I s o^{\otimes}\left(\omega_{z_{0}}^{(0)}, \omega_{z_{1}}^{(0)}\right)=\left\{(\gamma, \lambda) \in \mathbf{Z}^{\text {alg }} \mid \gamma(q) z_{0}=z_{1}\right\} .
$$


We thus obtain a local Galois groupoid at 0 , which we call $G^{(0)}$. Its base set is $\mathbf{C}^{*}$, the arrow sets $G^{(0)}(a, b)=I s o^{\otimes}\left(\omega_{a}^{(0)}, \omega_{b}^{(0)}\right)$ being described in the theorem. It is clearly connected on the base, so that we can take as local Galois group any group $G^{(0)}(a, a)$. The proalgebraic structure on each of the $G^{(0)}(a, b)$ is then induced by that of $\mathbf{Z}^{\text {alg }}$, of which they are Zariski closed subsets.

2.2.2. COROLLARY (the local Galois group). - With the same identifications,

$$
A u t^{\otimes}\left(\omega_{z_{0}}^{(0)}\right)=\left\{(\gamma, \lambda) \in \mathbf{Z}^{\text {alg }} \mid \gamma(q)=1\right\} .
$$

Let us now proceed to prove the theorem. Let $(\gamma, \lambda)$ be an element of $I s o^{\otimes}\left(\omega_{z_{0}}^{(0)}, \omega_{z_{1}}^{(0)}\right)$. Applied to the morphism $z:(\mathbf{C}, 1) \rightarrow(\mathbf{C}, q)$ in $\mathcal{P}^{(0)}$, it gives rise to the commutative diagram:

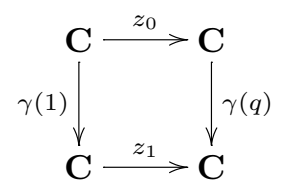

hence $\gamma(q) z_{0}=z_{1}$.

Conversely, suppose $\Phi=(\gamma, \lambda)$ with $\gamma(q) z_{0}=z_{1}$. We must check its compatibility with all morphisms in $\mathcal{P}^{(0)}$ (and not only in $\mathcal{R}$ ). That is, $F:\left(\mathbf{C}^{n}, A\right) \rightarrow\left(\mathbf{C}^{p}, B\right)$ being such a morphism, we want to show that the diagram:

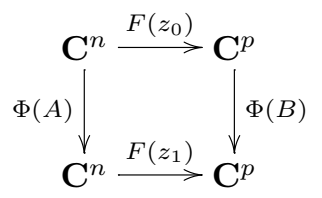

is commutative, where $z_{0}, z_{1} \in \mathbf{C}^{*}$ and $a z_{0}=z_{1}, a=\gamma(q)$. But we have $\left(\sigma_{q} F\right) A=B F$ thus, as noticed before, $\forall k \in \mathbf{Z}, F_{k}\left(q^{k} A\right)=B F_{k}$ so that $F_{k}: q^{k} A \rightarrow B$ is a morphism in $\mathcal{R}$. For this morphism we check the naturality of $\Phi$. Then:

$$
\begin{aligned}
F_{k} \Phi\left(q^{k} A\right) & =\Phi(B) F_{k} \\
\Rightarrow F_{k} a^{k} \Phi(A) & =\Phi(B) F_{k} \\
\Rightarrow F_{k} a^{k} z_{0}^{k} \Phi(A) & =\Phi(B) F_{k} z_{0}^{k} .
\end{aligned}
$$

Then, summing on $k$ gives:

$$
F\left(a z_{0}\right) \Phi(A)=\Phi(B) F\left(z_{0}\right)
$$

2.2.2.3. Incarnation of the local Galois groupoid. To incarnate $G^{(0)}$, one must show how its elements operate on an object $A$ of $\mathcal{E}_{f}^{(0)}$. So we take two base points $a, b \in \mathbf{C}^{*}$ and an element $(\gamma, \lambda) \in G^{(0)}(a, b)$. We must obtain an isomorphism from $\omega_{a}^{(0)}(A)$ to $\omega_{b}^{(0)}(A)$. Both vector spaces are equal to the space $\mathbf{C}^{n}$ underlying an object $A^{(0)}$ of $\mathcal{P}^{(0)}$ equivalent to $A$ (2.1.2.1). From 2.2.1, we see that the matrix describing the isomorphism we are looking for is $\gamma\left(A_{s}^{(0)}\right)\left(A_{u}^{(0)}\right)^{\lambda}$. Note that the semi-simple factor actually depends only on $\overline{A_{s}^{(0)}}$, not on $A_{s}^{(0)}$. 


\subsubsection{The local fundamental group}

We stick to our overloaded notation $G^{(0)}$ for the local Galois groupoid at 0 and at the same time for any of the local Galois groups, realized as:

$$
G^{(0)}(a, a) \simeq \operatorname{Hom}_{g r p}\left(\mathbf{C}^{*} / q^{\mathbf{Z}}, \mathbf{C}^{*}\right) \times \mathbf{C} .
$$

We shall now exhibit explicit elements in the local Galois group, aiming at a Zariski-dense finitely presented discrete group with a topological flavour. In the same way as the fundamental loop $1 \in \mathbf{Z} \subset \mathbf{Z}^{\text {alg }}=H_{\text {orm }}\left(\mathbf{C}^{*}, \mathbf{C}^{*}\right) \times \mathbf{C}$ splits into semi-simple and a unipotent parts, we shall consider separately the semi-simple and unipotent components of these elements. As for the latter, we shall take the obvious candidate: $1 \in \mathbf{C}$, which corresponds to the automorphism $\mathcal{X} \leadsto A_{u}^{(0)}$ of $\omega^{(0)}$. It generates a Zariski-dense subgroup of the unipotent component $G_{u}^{(0)}$. We concentrate hereafter on the semi-simple component $G_{s}^{(0)}$.

2.2.3.1. Explicit elements of $G_{s}^{(0)}$. The topological group $\mathbf{C}^{*} / q^{\mathbf{Z}}$ being compact, all the elements of $G_{s}^{(0)}$ built from continuous morphisms have their image in the biggest compact subgroup of $\mathbf{C}^{*}$, the unit circle $\mathbf{U}$. We now proceed to describe them.

We write $q=e^{-2 \imath \pi \tau}, \operatorname{Im}(\tau)>0$, whence the splitting $\mathbf{C}^{*}=\mathbf{U} \times q^{\mathbf{R}}$, where we put, for $y \in \mathbf{R}$, $q^{y}=e^{-2 \imath \pi \tau y}$ and $q^{\mathbf{R}}=\left\{q^{y} \mid y \in \mathbf{R}\right\}$. This comes (through the lift $\mathbf{C} \rightarrow \mathbf{C}^{*}, x \mapsto e^{2 \imath \pi x}$ ) from the splitting $\mathbf{C}=\mathbf{R} \oplus \mathbf{R} \tau$. We then define:

$$
\gamma_{1}:\left\{\begin{array}{l}
\mathbf{C}^{*} \rightarrow \mathbf{C}^{*}, \\
u q^{y} \mapsto u,
\end{array} \quad \gamma_{2}:\left\{\begin{array}{l}
\mathbf{C}^{*} \rightarrow \mathbf{C}^{*}, \\
u q^{y} \mapsto e^{2 \imath \pi y}
\end{array}\right.\right.
$$

More generally, one can define, for $b \in \mathbf{C}$, a group morphism $\gamma_{2}^{b}: u q^{y} \mapsto e^{2 \imath \pi b y}$. This will be used in 4.1.1.

2.2.3.2. LEMMA. - The subgroup of continuous elements of $G_{s}^{(0)}(a, a)$ is generated by $\gamma_{1}$ and $\gamma_{2}$.

All continuous group morphisms from $\mathbf{C}$ to $\mathbf{C}$ have the form $x+y \tau \mapsto \alpha x+\beta y$ with $\alpha, \beta \in \mathbf{C}$. Continuous group morphisms from $\mathbf{C}^{*}$ to $\mathbf{C}^{*}$ are obtained (through the same lifting as above) from those that send $\mathbf{Z}$ to itself, that is, those such that $\alpha \in \mathbf{Z}$. Such a morphism factors through $\mathbf{C}^{*} / q^{\mathbf{Z}}$ (i.e., it maps $q$ to 1 ) if and only if $\beta \in \mathbf{Z}$.

2.2.3.3. Lemma (a density criterion). - Let $\Gamma$ be a subset of $G_{s}^{(0)}=H_{\text {orm }}$ grp $\left(\mathbf{C}^{*} / \mathbf{q}^{\mathbf{Z}}, \mathbf{C}^{*}\right)$. Then $\Gamma$ generates a Zariski-dense subgroup of $G_{s}^{(0)}$ if and only if $\bigcap_{\gamma \in \Gamma} \operatorname{Ker} \gamma=\{1\}$.

By Chevalley's criterion, used in a similar way in [12] (p. 129, proof of Proposition 2.8; the precise formulation is given in loc. cit., first lecture, p. 40, Proposition 3.1.b and p. 41, Remark 3.2.a), the density condition amounts to the following: for any object $A$ of $\mathcal{E}_{f}^{(0)}$, if a line of $\omega^{(0)}(A)$ is stabilised by all $\gamma(A), \gamma \in \Gamma$, it is stabilised by all $\gamma(A), \gamma \in G_{s}^{(0)}$. This means that any common eigenvector of all the $\gamma\left(A_{s}^{(0)}\right), \gamma \in \Gamma$ is actually a common eigenvector of all the $\gamma\left(A_{s}^{(0)}\right), \gamma \in G_{s}^{(0)}$.

We now fix such an object $A$ and a non-zero $x \in \omega^{(0)}(A)=\mathbf{C}^{n}$. Up to conjugacy, one may assume that $A_{s}^{(0)}=\operatorname{diag}\left(c_{1}, \ldots, c_{n}\right)$. Then, $x$ is an eigenvector of $\gamma\left(A_{s}^{(0)}\right)$ if and only if, for any indices $i \neq j$ such that $x_{i} x_{j} \neq 0$ (let us say that such indices are linked), one has $\gamma\left(\overline{c_{i}}\right)=\gamma\left(\overline{c_{j}}\right)$ (recall from the introduction that $\bar{c}$ denotes the image of $c$ in $\mathbf{C}^{*} / \mathbf{q}^{\mathbf{Z}}$ ). Thus, the elements of $G_{s}^{(0)}$ whose action on $\omega^{(0)}(A)$ fixes the line $\mathbf{C} x$ are precisely those such that $\overline{c_{i} / c_{j}} \in \operatorname{Ker} \gamma$ for any pair of linked indices $(i, j)$. The lemma follows immediately. 
2.2.3.4. Two generating loops. According to the previous lemma, we are to choose elements of $\operatorname{Hom}_{\text {grp }}\left(\mathbf{C}^{*}, \mathbf{C}^{*}\right)$ sending $q$ to 1 and such that the intersection of their kernels is $q^{\mathbf{Z}}$. This cannot be done with one element, since $\mathbf{C}^{*} / q^{\mathbf{Z}}$ does not embed in $\mathbf{C}^{*}$ (for instance, because the former has 4 elements of order 2, while the latter has 2). To have anything explicit, we have to select among the morphisms found in 2.3.1. Since it is clear that $\operatorname{Ker} \gamma_{1} \cap \operatorname{Ker} \gamma_{2}=q^{\mathbf{z}}$, these two elements fit our needs. Note that $\gamma_{1}$ and $\gamma_{2}$ generate a free abelian group.

2.2.3.5. THEOREM. - The subgroup of $\mathrm{Hom}_{\text {grp }}\left(\mathbf{C}^{*}, \mathbf{C}^{*}\right) \times \mathbf{C}$ whose unipotent component is $\mathbf{Z} \subset \mathbf{C}$ and whose semi-simple component is generated by $\gamma_{1}$ and $\gamma_{2}$ is Zariski-dense in the local Galois group.

This subgroup can therefore be considered as the local fundamental group. As a consequence, all the semi-simple parts of our "monodromy matrices" will be unitary, in contrast to the classical (differential) case.

Since continuous elements of the Galois groupoid form a torsor under the action of any one of the groups just determined, it is enough, to get them all, to find one such element $g_{b, c}$ in each $G_{s}^{(0)}(b, c)$. To make it a groupoid, it is therefore enough to put $\Pi(a, b)=g_{a, b} \Pi(a, a)$, where $\Pi(a, a)$ is the group we just defined.

2.2.3.6. Corollary (the local fundamental groupoid).-Choose, for each $a \in \mathbf{C}^{*}, a$ logarithm $\alpha \in \frac{1}{2 \imath \pi} \log a$. Put $g_{a}=\gamma_{2}^{\alpha}$. One can then take $g_{b, c}=g_{\frac{c}{b}}$.

One cannot expect $g_{a}$ to be a continuous function of $a$, at the best, the choice of a logarithm involves a cut: the discontinuity at the cut will be interpreted in 2.2.4. There is also another interpretation of $g_{a}$ as an automorphism of the "field of solutions", corresponding to the translation $z \mapsto a z$ (in multiplicative notation) of the elliptic curve $\mathbf{E}_{q}$, see [31]. We postpone the geometrical interpretation of the local fundamental group and groupoid to 2.4.

\subsection{Flat vector bundles over the elliptic curve $\mathbf{E}_{q}$}

We give here a geometrical interpretation of $\mathcal{P}^{(0)}$, close to Weil's correspondence between flat vector bundles on a curve and representations of its fundamental group (see [37]).

Write $F i b(X)$ the category of holomorphic vector bundles over a compact Riemann surface $X$ and $F i b_{p}(X)$ the full subcategory of those which are flat, i.e. those whose transition matrices can be taken to be constant (vs holomorphic) for some adequate covering. Note that the morphisms between such flat bundles are not required to be constant.

\subsubsection{Holomorphic sections of a flat bundle over $\mathbf{E}_{q}$}

Let $A \in G l_{n}(\mathbf{C})$, thus an object of $\mathcal{P}^{(0)}$ as well as a linear $q$-difference system with constant coefficients. One introduces the equivalence relation $\sim_{A}$ on $\mathbf{C}^{*} \times \mathbf{C}^{n}$ generated by the relations:

$$
\forall(z, X) \in \mathbf{C}^{*} \times \mathbf{C}^{n}, \quad(z, X) \sim_{A}(q z, A X) .
$$

The first projection $\mathbf{C}^{*} \times \mathbf{C}^{n} \rightarrow \mathbf{C}^{*}$ is compatible with the action of $q^{\mathbf{Z}}$ on $\mathbf{C}^{*}$ and, factoring out, we define a holomorphic vector bundle of rank $n$ :

$$
F_{A} \stackrel{\text { def }}{=} \frac{\mathbf{C}^{*} \times \mathbf{C}^{n}}{\sim_{A}} \rightarrow \mathbf{E}_{q}=\frac{\mathbf{C}^{*}}{q^{\mathbf{Z}}}
$$

This is a particular case of Weil's correspondence alluded to above: the fundamental group of $\mathbf{E}_{q}$ is the lattice:

$$
\pi_{1}\left(\mathbf{E}_{q}\right)=\Lambda_{\tau}=\mathbf{Z}+\mathbf{Z} \tau
$$


where $q=e^{-2 \imath \pi \tau}$ and one takes the representation $\Lambda_{\tau} \rightarrow G l_{n}(\mathbf{C})$ sending 1 to $I_{n}$ and $\tau$ to $A$.

2.3.1.1. LEMMA. - There is a natural bijection between the solutions of the system with matrix A meromorphic on $\mathbf{C}$ and the holomorphic sections of $F_{A}$.

There is an obvious bijection between the holomorphic sections of $F_{A}$ and the solutions of the system with matrix $A$ holomorphic on $\mathbf{C}^{*}$. We have to show that the latter uniquely extend to meromorphic solutions on $\mathbf{C}$ (this, without having to prescribe growth conditions). We have already seen (2.1.3.2) that a solution meromorphic on $\mathbf{C}$ has to be holomorphic on $\mathbf{C}^{*}$ to prevent the accumulation of poles at 0 . So, let $F$ be holomorphic on $\mathbf{C}^{*}$ and such that $F(q z)=A F(z)$. Call $M$ the maximum of $\|F\|$ on the compact fundamental annulus $1 \leqslant|z| \leqslant|q|$ (this, for an arbitrary norm $\|-\|$ ) and, for $z \in \mathbf{C}^{*}$, put $k=\lfloor\ln |z| / \ln |q|\rfloor$ (where $\lfloor x\rfloor$ ) denotes the biggest integer less than or equal to $x$ ). One has:

$$
\left\|F\left(q^{-k} z\right)\right\| \leqslant M \Rightarrow\|F(z)\| \leqslant M\left\|\left.|| A\left|\left\|^{k} \Rightarrow\right\| F(z) \| \leqslant\right| z\right|^{\frac{\ln |||A| \| \mid}{\ln |q|}}\right.
$$

(|||- ||| the subordinate norm). This entails polynomial growth at 0 , hence a pole.

2.3.1.2. Remark. - For a unitary bundle, one can prove that all sections are actually constant $($ see $[37,34])$.

\subsubsection{Comparison of the categories $\mathcal{P}^{(0)}$ and $F i b_{p}\left(\mathbf{E}_{q}\right)$}

Since every morphism in $\mathcal{P}^{(0)}$ is holomorphic on $\mathbf{C}^{*}$, it defines a holomorphic map between the corresponding vector bundles and we clearly have a $\otimes$-functor from $\mathcal{P}^{(0)}$ to $F i b_{p}\left(\mathbf{E}_{q}\right)$.

\subsubsection{THEOREM. - This is a $\otimes$-equivalence.}

The full faithfulness comes from the existence of internal Hom in both categories, implying that the morphisms $A \rightarrow B$ (resp. $F_{A} \rightarrow F_{B}$ ) are in natural bijection with solutions of $\underline{\operatorname{Hom}}(A, B)$ (resp. $\left.\underline{\operatorname{Hom}}\left(F_{A}, F_{B}\right)\right)$ and from Lemma 2.3.1.1. Now, as regards essential surjectivity, let a vector bundle over $\mathbf{E}_{q}$ correspond to a representation of $\Lambda_{\tau}$ that maps 1 to $A$ and $\tau$ to $B$, these being commuting elements of $G l_{n}(\mathbf{C})$. One writes $A=e^{2 \imath \pi U}$ and uses the gauge transformation $e^{2 \imath \pi x U}$ to reduce this representation to one that sends 1 to $I_{n}$ (and $\tau$ to $A^{-\tau} B$ ). From the construction in 2.3.1, this comes from an object of $\mathcal{P}^{(0)}$.

\subsubsection{Fibre functors}

The category $\operatorname{Fib}_{p}\left(\mathbf{E}_{q}\right)$ is a thickening of $\operatorname{Rep}_{\mathbf{C}}\left(\pi_{1}\left(\mathbf{E}_{q}\right)\right)$ since it has the same objects but more morphisms: if $\phi: \pi_{1}\left(\mathbf{E}_{q}\right) \rightarrow G l(V)$ and $\psi: \pi_{1}\left(\mathbf{E}_{q}\right) \rightarrow G l(W)$ are two such representations, a morphism between the associated bundles gives rise to an equivariant morphism $\phi \rightarrow \psi$, that is, a holomorphic map $F: \mathbf{C} \rightarrow \mathcal{L}(V, W)$ such that:

$$
\forall \gamma \in \pi_{1}\left(\mathbf{E}_{q}\right), \quad \forall x \in \mathbf{C}, \quad \phi(\gamma) \circ F(x)=F(\gamma x) \circ \psi(\gamma) .
$$

This is a morphism in $\operatorname{Rep}_{\mathbf{C}}\left(\pi_{1}\left(\mathbf{E}_{q}\right)\right)$ if and only if $F$ is a constant map. Here, $\gamma$ operates on $x$ via the action of $\pi_{1}\left(\mathbf{E}_{q}\right)$ on the universal covering $\mathbf{C}$ of $\mathbf{E}_{q}$ (that is, the translation action of $\Lambda_{\tau}$ on $\mathbf{E}_{q}$ ).

2.3.3.1. Punctual fibre functors. Therefore, any fibre functor on $\mathcal{P}^{(0)}$ and $F i b_{p}\left(\mathbf{E}_{q}\right)$ naturally induces the usual fibre functor on $\operatorname{Rep}_{\mathbf{C}}\left(\pi_{1}\left(\mathbf{E}_{q}\right)\right)$ and, by the very same trick we already used in 2.1.3.4 and 2.2.2, we obtain $\operatorname{Gal}\left(F i b_{p}\left(\mathbf{E}_{q}\right)\right)$ as a proalgebraic subgroup of $\pi_{1}\left(\mathbf{E}_{q}\right)^{\text {alg }}$. The latter is isomorphic to $\mathbf{Z}^{\text {alg }} \oplus \mathbf{Z}^{\text {alg }}$. Actually, only one factor is really involved here since every bundle has been shown in 2.3.2.1 to be isomorphic to one on which 1 acts trivially (that is, one which already trivializes on the quotient covering $\mathbf{C}^{*}$ of the universal covering $\mathbf{C}$ ). 
2.3.3.2. The global fibre functor. On the other hand, the equivalence of $\mathcal{P}^{(0)}$ with $F i b_{p}\left(\mathbf{E}_{q}\right)$ may itself be viewed as a fibre functor on $\mathcal{E}_{f}^{(0)}$ in the following way. Call $S$ the unique curve (scheme) over $\mathbf{C}$ with underlying analytic space $S^{a n}=\mathbf{E}_{q}$. Then, holomorphic vector bundles over $\mathbf{E}_{q}$ "are" locally free sheaves and we get a fibre functor over $S$ in the sense of [11]. The Theorem 1.12 of loc. cit. then provides us with a groupoid structure over $S$ :

2.3.3.3. THEOREM. - The tensor category $\mathcal{E}_{f}^{(0)}$ is equivalent to the category of representations of a groupoid $G^{(0)}$ that is faithfully flat over $S \times S$.

In essence, this says that $G^{(0)}$ acts transitively on the base $S$ and that composition of paths is a morphism with respect to the proalgebraic structure on each $G^{(0)}(a, b)$, but also with respect to the algebraic structure on the base $S$.

\subsubsection{The classification theorem of Baranovsky and Ginzburg}

Let $G$ denote a complex algebraic group and write $G((z))$ for the group of $\mathbf{C}((z))$ rational points of $G$, where $\mathbf{C}((z))$ is the field of formal Laurent series, a kind of "loop group". Write $G \llbracket z \rrbracket$ for its subgroup of $\mathbf{C} \llbracket z \rrbracket$ rational points. Then define a "twisted" conjugation action of $G((z))$ on itself by putting, for $a(z), g(z) \in G((z))$ :

$$
{ }^{g} a(z)=g(q \cdot z) \cdot a(z) \cdot g(z)^{-1} .
$$

This twisted conjugation action can actually be seen as a plain conjugation action in some larger group: putting $a(z) \mapsto a(t . z)$ defines a $\mathbf{C}^{*}$-action on the loop group $G((z))$ (the "rotation of the loop"), hence a semi-direct product, and twisted conjugacy classes are actually ordinary conjugacy classes in a Kac-Moody group.

The following is stated and proved in [3]:

THEOREM. - If $G$ is connected and semi-simple, there is a natural bijection between the set of integral twisted conjugacy classes in $G((z))$ and the set of isomorphism classes of semi-stable holomorphic principal $G$-bundles on the elliptic curve $E=\mathbf{C}^{*} / q^{\mathbf{Z}}$.

Here, integral twisted conjugacy classes are those which contain an element of $G \llbracket z \rrbracket$ : they are analogous to our fuchsian equations. One subtlety of this theorem (and the main difficulty in its proof) is that it provides an analytic classification of formal objects. The authors give a tannakian extension of this theorem. They define a tensor category $\mathcal{M}_{q}$ of formal integral $q$-difference modules and prove:

THEOREM. - It is equivalent to the tensor category of degree zero semi-stable holomorphic vector bundles on $E$.

In the electronic (IMRN) version of their paper, they moreover quote a computation by Kontsevich of the corresponding Galois group: the result is the same as our Corollary 2.2.2.2.

\subsection{Heuristic topological interpretation and perspectives}

\subsubsection{A "local" elliptic curve}

Recall that our constructions aim at a geometric understanding of $q$-difference equations. Extending the class of morphisms of the category $\mathcal{E}_{f}$ as we did in 2.1 amounts to a localisation for the transcendental topology. Accordingly, our vector bundles on $\mathbf{E}_{q}$ can be considered as induced by equivariant vector bundles on the germ of complex space $\left(\mathbf{C}^{*}, 0\right)$ and the curve $\mathbf{E}_{q}$ itself as the quotient of the germ $\left(\mathbf{C}^{*}, 0\right)$ by the action of the dilatation $\sigma_{q}$. We visualize this curve as "local 
at $0 \in \mathbf{S}$ ", since it carries the local geometry of fuchsian q-difference equations. The loops found in 2.2.3 can been interpreted as the two fundamental loops of $\mathbf{E}_{q}$. They are algebraically free and generate a free abelian group of rank 2 . However, they satisfy a "transcendental relation", as predicted in [23]:

$$
\gamma_{1} \gamma_{2}^{-\tau}=I d_{\mathbf{C}^{*}},
$$

the semi-simple fundamental loop of $\mathbf{Z}^{\text {alg }}$. Suppose the logarithms involved in the definition of the $g_{a}$ have been chosen continuously, but for some cut: for instance, the main determination (alternatively, see 3.2.2). Let $a$ turn counterclockwise once around 0 . Then $g_{a}\left(u q^{y}\right)=e^{-2 \imath \pi \tau \alpha y}$ is multiplied by $e^{-2 \imath \pi y}$, that is, by $\gamma_{2}\left(u q^{y}\right)$. This means that $\gamma_{2}$ represents the plain classical loop around 0 in $\mathbf{C}^{*}$. This can be seen yet another way: suppose we change our choice of a logarithm of $q$, writing $q=e^{-2 \imath \pi \tau^{\prime}}$, where $\tau^{\prime}=\tau+m, m \in \mathbf{Z}$. Then, the formulas in 2.2.3.1 produce modified loops $\gamma_{1}^{\prime}$ and $\gamma_{2}^{\prime}$ and one checks that $\gamma_{1}^{\prime}=\gamma_{1} \gamma_{2}^{m}$ and $\gamma_{2}^{\prime}=\gamma_{2}$. To interpret $\gamma_{1}$ as the second generating loop of $\mathbf{E}_{q}$ is not so easy while staying in a strict local context. It will be seen in 3.2.2.2 to be the defect of ellipticity of the twisted connection matrix, which is the generic Galois isomorphism linking 0 to $\infty$. This suggests the interpretation of $\gamma_{1}$ as the beginning of the movement from 0 to $\infty$ along a $q$-spiral, or even a precession.

\subsubsection{Irregular equations and "infinitesimal" elliptic curves}

The next step in our program is to tackle the irregular local theory. The classification problem is solved in a common work with Jean-Pierre Ramis and Changgui Zhang (see [25]). It uses a new discrete summation method for divergent solutions (see [38]), the existence of a canonical filtration by the slopes for $q$-difference modules (see [28,29] and [32]) and sheaf theoretic methods due to Malgrange, Martinet and Ramis. The latter allow us to extend and enrich the geometric setting of the present paper. In [25], we use a dynamical interpretation (due to J.-L. Martins) of classical asymptotical developments. This version can be discretized in the following sense. While the sheaf of Malgrange (see , for instance, [7]) is defined on the horizon $\mathbf{S}^{1}=\mathbf{C}^{*} / \Sigma$ of the action of the semigroup $\left.\Sigma=\right] 0 ; 1$, taking instead the semigroup $\Sigma_{q}=q^{-\mathbf{N}}$, we get the horizon $\mathbf{C}^{*} / \Sigma_{q}$, whence a sheaf defined on the elliptic curve $\mathbf{E}_{q}$. Our vector bundles are related to this sheaf in the fuchsian case. The elliptic curve $\mathbf{E}_{q}$ could also be viewed as the quotient of an infinitesimal neighborhood of 0, predicted by Ramis in [23]. Actually, as shown in [25], there is a whole family of such infinitesimal neighborhoods related to all the possible slopes and the corresponding sheaves of functions). The results presented here can easily be extended to the category of "tamely irregular modules"; these are direct sums of pure modules. One thus computes a Galois group $G_{m i}^{(0)}$. Then, the graded functor $g r$ associated to the canonical filtration being faithful, exact and $\otimes$-preserving, one realizes the general local Galois group $G_{i}^{(0)}$ as a semi-direct product of $G_{m i}^{(0)}$ by a pro-unipotent group, generated by the Stokes operators. This will be detailed in [30].

\section{Global theory}

\subsection{The global Galois groupoid}

\subsubsection{Birkhoff's classification revisited}

We shall give a galoisian meaning to Birkhoff's classification theorem.

3.1.1.1. The category $\mathcal{C}$ of connection data. We introduce a categorical variant $\mathcal{C}$ of the set of classifying data introduced in 1.3.3. The objects are triples $\left(A^{(0)}, M, A^{(\infty)}\right)$ where, for some 
$n \in \mathbf{N}^{*}, A^{(0)}, A^{(\infty)} \in G l_{n}(\mathbf{C}), M \in G l_{n}\left(\mathcal{M}\left(\mathbf{C}^{*}\right)\right)$ and moreover:

$$
\left(\sigma_{q} M\right) A^{(0)}=A^{(\infty)} M
$$

Said otherwise, $M: A^{(0)} \rightarrow A^{(\infty)}$ is an isomorphism in $\mathcal{E}_{f}^{(*)}$. Morphisms

$$
\left(A^{(0)}, M, A^{(\infty)}\right) \rightarrow\left(B^{(0)}, N, B^{(\infty)}\right)
$$

are pairs $\left(S^{(0)}, S^{(\infty)}\right)$ such that:

$$
\left\{\begin{array}{l}
S^{(0)}: A^{(0)} \rightarrow B^{(0)} \text { is a morphism in } \mathcal{E}_{f}^{(0)} \text { or, what amounts to the same, in } \mathcal{P}^{(0)} \\
S^{(\infty)}: A^{(\infty)} \rightarrow B^{(\infty)} \text { is a morphism in } \mathcal{E}_{f}^{(\infty)} \text { or, what amounts to the same, in } \mathcal{P}^{(\infty)}
\end{array}\right.
$$

and, moreover, the following square commutes:

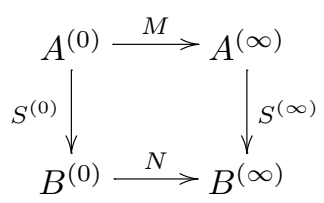

In the same vein as in 2.3 , objects of $\mathcal{C}$ can be interpreted as triples $\left(F^{(0)}, f, F^{(\infty)}\right)$ where $\left(F^{(0)}, F^{(\infty)}\right)$ are holomorphic vector bundles over $\mathbf{E}_{q}$ and $f: F^{(0)} \rightarrow F^{(\infty)}$ is a meromorphic map between them.

Then, we make $\mathcal{C}$ a tensor category by endowing it with the natural (componentwise) tensor product; here, we use the conventions of 1.1.2 for the tensor product of matrices. The resulting category is plainly an abelian $\mathbf{C}$-linear neutral tannakian category. Moreover, after [12], Proposition 2.21, the projections to $\mathcal{E}_{f}^{(0)}$ and $\mathcal{E}_{f}^{(\infty)}$ induce closed embeddings of $G^{(0)}$ and $G^{(\infty)}$ into the Galois group of $\mathcal{C}$. Our goal is to build an equivalence of $\mathcal{C}$ with $\mathcal{E}_{f}$.

3.1.1.2. The category $\mathcal{S}$ of solutions. In Birkhoff's method, one encodes a fuchsian equation $\sigma X=A X$, by its local solutions at 0 and $\infty$ :

$$
X^{(0)}=M^{(0)} e_{q, A^{(0)}} \quad \text { and } \quad X^{(\infty)}=M^{(\infty)} e_{q, A^{(\infty)}} .
$$

In more intrinsic terms, we shall use the local flat forms $A^{(0)}$ and $A^{(\infty)}$ together with the meromorphic gauge transformations $M^{(0)}$ (reducing $A$ to $A^{(0)}$ ) and $M^{(\infty)}$ (reducing $A$ to $A^{(\infty)}$ ). Due to the non canonicity of all these local data (except from the generic case of strictly fuchsian non resonant equations), we shall eventually map solutions to equations and not the other way round.

We shall therefore consider local pairs at 0 and at $\infty$ :

$$
\begin{aligned}
\left(A^{(0)}, M^{(0)}\right) & \in G L_{n}(\mathcal{M}(\mathbf{C})) \times G L_{n}(\mathbf{C}), \\
\left(A^{(\infty)}, M^{(\infty)}\right) & \in G L_{n}\left(\mathcal{M}\left(\mathbf{C}_{\infty}\right)\right) \times G L_{n}(\mathbf{C}) .
\end{aligned}
$$

We shall say that two such pairs are connected if one of the following (obviously) equivalent conditions is realized: 
1. The following two expressions are equal:

$$
\left(\sigma M^{(\infty)}\right) A^{(\infty)}\left(M^{(\infty)}\right)^{-1}=\left(\sigma M^{(0)}\right) A^{(0)}\left(M^{(0)}\right)^{-1} .
$$

2. The matrix $M=\left(M^{(\infty)}\right)^{-1} M^{(0)} \in G L_{n}\left(\mathcal{M}\left(\mathbf{C}^{*}\right)\right)$ is such that $(\sigma M) A^{(0)}=A^{(\infty)} M$.

In this case, we shall call $A$ the common value of the two expressions appearing in the first condition. Being meromorphic on both $\mathbf{C}$ and $\mathbf{C}_{\infty}$, it is meromorphic on $\mathbf{S}$, that is, rational: $A \in G L_{n}(\mathbf{C}(z))$. Moreover, $M^{(0)}$ (resp. $\left.M^{(\infty)}\right)$ can be viewed as a morphism from $A^{(0)}$ (resp. from $\left.A^{(\infty)}\right)$ to $A$.

We now define our category of solutions.

- Objects of $\mathcal{S}$. They are the quadruples:

$$
\left(A^{(0)}, M^{(0)}, A^{(\infty)}, M^{(\infty)}\right) \in G l_{n}(\mathbf{C}) \times G l_{n}(\mathcal{M}(\mathbf{C})) \times G l_{n}(\mathbf{C}) \times G l_{n}\left(\mathcal{M}\left(\mathbf{C}_{\infty}\right)\right)
$$

such that the two component pairs $\left(A^{(0)}, M^{(0)}\right)$ and $\left(A^{(\infty)}, M^{(\infty)}\right)$ are connected.

- Morphisms in $\mathcal{S}$. The morphisms from $\left(A^{(0)}, M^{(0)}, A^{(\infty)}, M^{(\infty)}\right)$ to $\left(B^{(0)}, N^{(0)}, B^{(\infty)}\right.$, $\left.N^{(\infty)}\right)$ in $\mathcal{S}$ are the triples $\left(F, S^{(0)}, S^{(\infty)}\right)$ such that

$$
\left\{\begin{array}{l}
S^{(0)}: A^{(0)} \rightarrow B^{(0)} \text { is a morphism in } \mathcal{E}_{f}^{(0)}, \\
S^{(\infty)}: A^{(\infty)} \rightarrow B^{(\infty)} \text { is a morphism in } \mathcal{E}_{f}^{(\infty)}
\end{array}\right.
$$

and, moreover, the following squares commute:

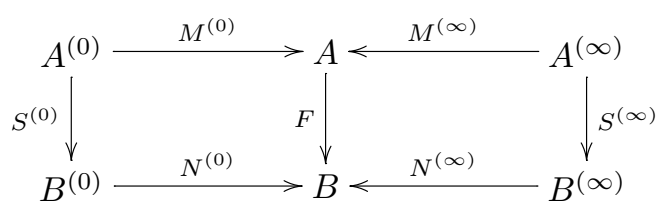

Here, $A$ and $B$ are defined according to our previous convention. One then notes, first that

$$
F=N^{(\infty)} S^{(\infty)}\left(M^{(\infty)}\right)^{-1}=N^{(0)} S^{(0)}\left(M^{(0)}\right)^{-1}
$$

is meromorphic on both $\mathbf{C}$ and $\mathbf{C}_{\infty}$, therefore rational; second, that, by any of these two expressions for $F,(\sigma F) A=B F$, that is, $F$ is a morphism $A \rightarrow B$ in $\mathcal{E}_{f}$.

- Tensor structure on $\mathcal{S}$. The tensor product of objects (resp. morphisms) is defined componentwise on the quadruples (resp. triples), using the usual identifications.

Again, one has obtained an abelian $\mathbf{C}$-linear neutral tannakian category such that the projections to $\mathcal{E}_{f}^{(0)}$ and $\mathcal{E}_{f}^{(\infty)}$ induce closed embeddings of $G^{(0)}$ and $G^{(\infty)}$ into the Galois group of $\mathcal{S}$.

3.1.1.3. Proposition (equivalence of the tensor categories $\mathcal{E}_{f}, \mathcal{S}$ and $\mathcal{C}$ ). - One keeps the previous conventions for $A$ and $M$. Then, taking:

$$
\left\{\begin{array}{l}
\left(A^{(0)}, M^{(0)}, A^{(\infty)}, M^{(\infty)}\right) \sim A, \\
\left(F, S^{(0)}, S^{(\infty)}\right) \sim F
\end{array}\right.
$$

and

$$
\left\{\begin{array}{l}
\left(A^{(0)}, M^{(0)}, A^{(\infty)}, M^{(\infty)}\right) \leadsto\left(A^{(0)}, M, A^{(\infty)}\right), \\
\left(F, S^{(0)}, S^{(\infty)}\right) \sim\left(S^{(0)}, S^{(\infty)}\right)
\end{array}\right.
$$

provides us with $\mathbf{C}$-linear $\otimes$-equivalences from $\mathcal{S}$ to $\mathcal{E}_{f}$ and from $\mathcal{S}$ to $\mathcal{C}$. 
It is clear that one has defined two $\otimes$-functors and that the first one is fully faithful. That it is also essentially surjective stems from the existence of flat local reductions at 0 and at $\infty$ for any fuchsian equation, as recalled in 1.2.

That the second functor is fully faithful comes from the fact that the equalities:

$$
F=N^{(\infty)} S^{(\infty)}\left(M^{(\infty)}\right)^{-1}=N^{(0)} S^{(0)}\left(M^{(0)}\right)^{-1}
$$

give a unique antecedent to a pair $\left(S^{(0)}, S^{(\infty)}\right)$. For essential surjectivity, one starts from an object $\left(A^{(0)}, M, A^{(\infty)}\right)$ of $\mathcal{C}$. Since $M \in G L_{n}\left(\mathcal{M}\left(\mathbf{C}^{*}\right)\right)$, Birkhoff's lemma (see [27], 2.2 and 2.3) allows us to write:

$$
M=\left(M^{(\infty)}\right)^{-1} M^{(0)}, \quad \text { where }\left(M^{(0)}, M^{(\infty)}\right) \in G l_{n}(\mathcal{M}(\mathbf{C})) \times G l_{n}\left(\mathcal{M}\left(\mathbf{C}_{\infty}\right)\right) .
$$

It is then clear that $\left(A^{(0)}, M^{(0)}, A^{(\infty)}, M^{(\infty)}\right)$ is a connected quadruple and an antecedent of $\left(A^{(0)}, M, A^{(\infty)}\right)$.

3.1.1.4. Singularities and exponents. Let $\Sigma$ be a finite subset of $\mathbf{C}^{*}$. We shall have to consider the full subcategory $\mathcal{C}_{\Sigma}$ of $\mathcal{C}$ whose objects are the triples $\left(A^{(0)}, M, A^{(\infty)}\right)$ such that $\mathcal{S}(M) \subset q^{\mathbf{z}} \Sigma$. It is stable by all tensor and abelian constructions, hence a tannakian subcategory. From 1.2.5 and from the remarks in loc. cit., 2.3.1, one draws that the equivalence shown in 3.1.1.3 induces an equivalence of the tannakian categories $\mathcal{E}_{f, \Sigma}$ and $\mathcal{C}_{\Sigma}$.

\subsubsection{The global Galois groupoid and the global fundamental groupoid}

Composing the above projections with fibre functors $\omega_{z_{0}}^{(0)}, \omega_{z_{0}}^{(\infty)}$ provides us with two fibre functors on $\mathcal{C}$. We shall call these restrictions by the same names.

3.1.2.1. Definition. - The Galois groupoid of $\mathcal{C}$ is the groupoid $G$ having as base set:

$$
\left\{\omega_{a}^{(0)} \mid a \in \mathbf{C}^{*}\right\} \cup\left\{\omega_{a}^{(\infty)} \mid a \in \mathbf{C}^{*}\right\},
$$

and such that, for any two $a, b \in \mathbf{C}^{*}$ :

$$
\begin{aligned}
G\left(\omega_{a}^{(0)}, \omega_{b}^{(0)}\right) & =I s 0^{\otimes}\left(\omega_{a}^{(0)}, \omega_{b}^{(0)}\right), \\
G\left(\omega_{a}^{(\infty)}, \omega_{b}^{(\infty)}\right) & =I s 0^{\otimes}\left(\omega_{a}^{(\infty)}, \omega_{b}^{(\infty)}\right), \\
G\left(\omega_{a}^{(0)}, \omega_{b}^{(\infty)}\right) & =\left\{\begin{array}{l}
\text { if } a=b: \operatorname{Iso}{ }^{\otimes}\left(\omega_{a}^{(0)}, \omega_{b}^{(\infty)}\right), \\
\text { if } a \neq b: \emptyset .
\end{array}\right.
\end{aligned}
$$

The local groupoids computed in Section 2 embed in the corresponding subgroupoids of $G$, giving many elements in the groups $A u t^{\otimes}\left(\omega_{z_{0}}^{(0)}\right)$ and $A u t^{\otimes}\left(\omega_{z_{0}}^{(\infty)}\right)$ for all $z_{0} \in \mathbf{C}^{*}$ and in the sets $I s o^{\otimes}\left(\omega_{z_{0}}^{(0)}, \omega_{z_{1}}^{(0)}\right)$ and $I s o^{\otimes}\left(\omega_{z_{0}}^{(\infty)}, \omega_{z_{1}}^{(\infty)}\right)$ for all $z_{0}, z_{1} \in \mathbf{C}^{*}$. To complete this and connect $G$, we want to build sufficiently many elements in the sets $I s o^{\otimes}\left(\omega_{z_{0}}^{(0)}, \omega_{z_{0}}^{(\infty)}\right)$ for all $z_{0} \in \mathbf{C}^{*}$. For instance, one gets such a $\otimes$-isomorphism from $\omega_{z_{0}}^{(0)}$ to $\omega_{z_{0}}^{(\infty)}$ by taking $\left(A^{(0)}, M, A^{(\infty)}\right)$ to $M$. But this is not defined over $\mathbf{C}^{*}$, so we change our way. This can be done by evaluating $M$ at points $z_{0} \notin \mathcal{S}(M)$. For that, we fix a finite subset $\Sigma$ of $\mathbf{C}^{*}$ and restrict to the full subcategory $\mathcal{C}_{\Sigma}$ of $\mathcal{C}$.

\subsubsection{Proposition. - For any such point $z_{0}$, the natural transformation}

$$
\Gamma_{z_{0}}:\left(A^{(0)}, M, A^{(\infty)}\right) \sim M\left(z_{0}\right)
$$

is an element of $I s o^{\otimes}\left(\omega_{z_{0}}^{(0)}, \omega_{z_{0}}^{(\infty)}\right)$.

$4^{\mathrm{e}}$ SÉRIE - TOME $36-2003-\mathrm{N}^{\circ} 6$ 
Here and in the following, we keep the same names for the restrictions to $\mathcal{C}_{\Sigma}$ of the fibre functors $\omega_{z_{0}}^{(0)}, \omega_{z_{0}}^{(\infty)}\left(z_{0} \notin q^{\mathbf{Z}} \Sigma\right)$. The proof of the proposition is then more or less tautological. Tensor preservation comes from the definition of the tensor structure componentwise, plus the obvious fact that $\left(M \otimes M^{\prime}\right)\left(z_{0}\right)=M\left(z_{0}\right) \otimes M^{\prime}\left(z_{0}\right)$. Functoriality comes from the commuting square in the definition of morphisms in 3.1.1.1 plus the obvious computation:

$$
N\left(z_{0}\right) S^{(0)}\left(z_{0}\right)=\left(N S^{(0)}\right)\left(z_{0}\right)=\left(S^{(\infty)} M\right)\left(z_{0}\right)=S^{(\infty)}\left(z_{0}\right) M\left(z_{0}\right) .
$$

We see $\Gamma_{z_{0}}$ as a path connecting the points $\omega_{z_{0}}^{(0)}, \omega_{z_{0}}^{(\infty)}$ of the groupoid $G$.

3.1.2.3. THEOREM. - The local groupoids at 0 and at $\infty$ (defined and computed in Section 2) together with the paths $\Gamma_{z_{0}}, z_{0} \notin \Sigma$ generate a Zariski-dense subgroupoid of the Galois groupoid of $\mathcal{C}_{\Sigma}$.

We appeal again to the criterion of Chevalley (see the proof of 2.2.3.3). It can easily be extended to the case of a groupoid in the following way. We choose an object

$$
\mathcal{X}=\left(A^{(0)}, M, A^{(\infty)}\right)
$$

and, for each basepoint $\omega_{a}^{(0)}$ (resp. $\left.\omega_{a}^{(\infty)}\right), a \notin q^{\mathbf{z}} \Sigma$, a line $D_{a}^{(0)} \subset \omega_{a}^{(0)}(\mathcal{X})$ (resp. $D_{a}^{(\infty)} \subset$ $\omega_{a}^{(\infty)}(\mathcal{X})$ ) and we assume that this family of lines is globally stable under the action of $G^{(0)}$, $G^{(\infty)}$ and our special paths. It is then sufficient to check that this family of lines is actually stable under the action of the whole Galois groupoid.

By Tannaka duality for the category $\mathcal{E}_{f}^{(0)}$ and for the groupoid $G^{(0)}$, we see that the family of lines $D_{a}^{(0)}$ induces a subrepresentation of rank 1 of the representation defined by the object $A^{(0)}$, hence comes from a subobject of rank 1 of $A^{(0)}$. This subobject is an injection $x^{(0)}: a^{(0)} \rightarrow A^{(0)}$ in $\mathcal{E}_{f}^{(0)}$ and we may take it to lie in $\mathcal{P}^{(0)}$. This means that $a^{(0)} \in \mathbf{C}^{*}$, that $x^{(0)}$ is a function holomorphic on $\mathbf{C}^{*}$ and that, for all $a \notin q^{\mathbf{z}} \Sigma$, the line $D_{a}^{(0)}$ is the image of the linear map $\omega_{a}^{(0)}\left(x^{(0)}\right)$, that is: $D_{a}^{(0)}=\mathbf{C} x^{(0)}(a)$.

The same argument on the $\infty$ side shows that there exists a subobject $x^{(\infty)}: a^{(\infty)} \rightarrow A^{(\infty)}$ such that, for all $a \notin q^{\mathbf{z}_{\Sigma}}, D_{a}^{(\infty)}=\mathbf{C} x^{(\infty)}(a)$. The condition of stability under our special paths says that $\forall a \notin q^{\mathbf{z}_{\Sigma}, M(a) D_{a}^{(0)}}=D_{a}^{(\infty)}$, so that, out of $q^{\mathbf{z}_{\Sigma}}$, there exists a holomorphic function $m$ such that $M(a) x^{(0)}(a)=m(a) x^{(\infty)}(a)$. This amounts to say that $\phi=\left(x^{(0)}, x^{(\infty)}\right)$ is a morphism from $\mathcal{X}^{\prime}=\left(a^{(0)}, m, a^{(\infty)}\right)$ to $\mathcal{X}=\left(A^{(0)}, M, A^{(\infty)}\right)$ in $\mathcal{C}$.

We now take an arbitrary galoisian isomorphism, that is, an element $h \in I s o^{\otimes}\left(\omega_{a}^{(0)}, \omega_{a}^{(\infty)}\right)$. The functoriality condition gives rise to a commutative diagram:

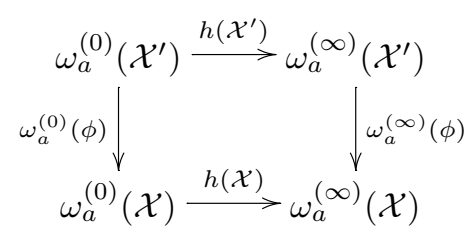

Then $h(\mathcal{X}) x^{(0)}(a)=h\left(\mathcal{X}^{\prime}\right) x^{(\infty)}(a)$; since $h\left(\mathcal{X}^{\prime}\right) \in \mathbf{C}^{*}$, this shows the stability of our family of lines under the action of $I s o^{\otimes}\left(\omega^{(0)}, \omega^{(\infty)}\right)$, hence also under the action of the whole Galois groupoid. 


\subsubsection{The case of a regular equation}

3.1.3.1. Regular triples. Let us now consider the case of a regular equation. Recall from Section 1 that $A$ is said to be regular at 0 (resp. at $\infty$ ) if it is equivalent to the identity matrix $I_{n}$ at 0 (resp. at $\infty$ ). Then $A$ has a local reduction at $0, M^{(0)}: I_{n} \rightarrow A$ as well as a local reduction at $\infty, M^{(\infty)}: I_{n} \rightarrow A$. One can therefore associate to $A$ the triple $\left(I_{n}, M, I_{n}\right)$ in $\mathcal{C}_{\Sigma}$, with $\Sigma=\mathcal{S}(A), M=M=\left(M^{(\infty)}\right)^{-1} M^{(0)}$. Moreover, in this case, $M$ is "the" connection matrix $P$ and it is elliptic.

3.1.3.2. COROLLARY (the Galois group of a regular equation). - The Galois group at any point $\omega_{z_{0}}^{(0)}$ is the Zariski closure of the subgroup generated by the values $(P(a))^{-1} P(b)$ for $a, b \notin q^{\mathbf{Z}} \Sigma$.

Indeed, from the equalities $A^{(0)}=A^{(\infty)}=I_{n}$, we draw that the local Galois groupoids at 0 and at $\infty$ of the equation are trivial (there are only identity arrows between any two points). The conclusion now follows from Theorem 3.1.2.3.

This is the case tackled by Etingof in [14], and this proposition is his main result.

\subsection{Birkhoff's method}

We shall follow here Birkhoff's method more closely, using the connection matrix $P$ itself (together with local linear data) to encode fuchsian equations, then, trying to interpret its values as monodromy data. However, the bad multiplicative properties of any canonical choice of solutions, hence of the matrix $P$ itself, lead us to twist first the tensor product in the category of connection data, second the connection matrix itself into a matrix $\breve{P}$ in order to get galoisian properties. The relation with the point of view of 3.1 is explained in 3.2.3. Proofs and details can be found in [26] and [31].

\subsubsection{Equivalences of tannakian categories}

We encode a fuchsian equation $\sigma X=A X$ by its local solutions at 0 and $\infty$ :

$$
X^{(0)}=M^{(0)} e_{q, A(0)} \quad \text { and } \quad X^{(\infty)}=M^{(\infty)} e_{q, A(\infty)}
$$

and its connection matrix $P=\left(X^{(\infty)}\right)^{-1} X^{(0)}$, as defined in 1.3.3. We shall consistently use these notations herebelow, without further notice. These data are not unique, so that we use an intermediate category of solutions to link equations and connection triples. By necessity, one does not take the natural tensor product $X_{1}^{(0)} \otimes X_{2}^{(0)}$ on solutions. This comes from the fact that $e_{q, A} \otimes e_{q, B} \neq e_{q, A \otimes B}$, thereby destroying our normal forms for solutions. The defect of equality has been analyzed in 1.2.2.3. We thus give a special notation for the twisted tensor product:

$$
X_{1}^{(0)} \underline{\otimes} X_{2}^{(0)}=\left(M_{1}^{(0)} \otimes M_{2}^{(0)}\right) e_{q, A_{1}^{(0)} \otimes A_{2}^{(0)}},
$$

and similarly at $\infty$. This is related to the natural tensor product in the following way:

$$
X_{1}^{(0)} \underline{\otimes} X_{2}^{(0)}=\left(X_{1}^{(0)} \otimes X_{2}^{(0)}\right) \Phi\left(A_{1}^{(0)}, A_{2}^{(0)}\right) .
$$

By necessity, one is thus led to twist the natural tensor product of connection matrices:

$$
P_{1} \otimes P_{2}=\left(X_{1}^{(\infty)} \otimes X_{2}^{(\infty)}\right)^{-1}\left(X_{1}^{(0)} \otimes X_{2}^{(0)}\right)
$$


in the following way:

$$
P_{1} \otimes P_{2}=\Phi\left(A_{1}^{(\infty)}, A_{2}^{(\infty)}\right)\left(P_{1} \otimes P_{2}\right)\left(\Phi\left(A_{1}^{(0)}, A_{2}^{(0)}\right)\right)^{-1} .
$$

This notation is slightly ambiguous, since the right hand side does not really depend on $P_{1}$ and $P_{2}$ alone, but also involves the local linear data $A_{1}^{(0)}, A_{2}^{(0)}, A_{1}^{(\infty)}, A_{2}^{(\infty)}$. Note that, in the case that one of $A_{1}^{(0)}, A_{2}^{(0)}$ is unipotent and the same holds at $\infty$, we have $P_{1} \underline{\otimes} P_{2}=P_{1} \otimes P_{2}$. This is the case, e.g. for regular equations (i.e. such that the matrices at 0 and $\infty$ are $I_{n}$ ).

3.2.1.1. The category $\mathcal{S}^{\prime}$ of solutions. Its objects are quadruples

$$
\left(A^{(0)}, M^{(0)}, A^{(\infty)}, M^{(\infty)}\right) \in G l_{n}(\mathbf{C}) \times G l_{n}(\mathcal{M}(\mathbf{C})) \times G l_{n}(\mathbf{C}) \times G l_{n}\left(\mathcal{M}\left(\mathbf{C}_{\infty}\right)\right)
$$

such that $X^{(0)}=M^{(0)} e_{q, A(0)}$ and $X^{(\infty)}=M^{(\infty)} e_{q, A(\infty)}$ are connected in a sense similar to 3.1.1.2. One can likewise adapt the definitions in such a way as to get a neutral tannakian category.

3.2.1.2. The category $\mathcal{C}^{\prime}$ of connection data. Birkhoff's classification theorem (see [5,27]) amounts to saying that the data $\left(A^{(0)}, P, A^{(\infty)}\right)$ are enough to recover $A$ up to rational equivalence. We shall now give a categorical formulation of it.

- Objects of $\mathcal{C}^{\prime}$. They are the triples:

$$
\left(A^{(0)}, P, A^{(\infty)}\right) \in G l_{n}(\mathbf{C}) \times G l_{n}\left(\mathcal{M}\left(\mathbf{E}_{q}\right)\right) \times G l_{n}(\mathbf{C}) .
$$

- Morphisms in $\mathcal{C}^{\prime}$. The morphisms from the object $\left(A^{(0)}, P, A^{(\infty)}\right)$ of order $n$ to the object $\left(B^{(0)}, Q, B^{(\infty)}\right)$ of order $p$ are the pairs

$$
\left(R^{(0)}, R^{(\infty)}\right) \in M_{p, n}(\mathbf{C}) \times M_{p, n}(\mathbf{C})
$$

such that

$$
\left\{\begin{array}{l}
R^{(0)} \overline{A^{(0)}}=\overline{B^{(0)}} R^{(0)} \\
R^{(\infty)} P=Q R^{(0)} \\
R^{(\infty)} \overline{A^{(\infty)}}=\overline{B^{(\infty)}} R^{(\infty)}
\end{array}\right.
$$

This can be justified by the properties proved in 1.2.4 (for more details, see [26]). Note the following consequences of the definition:

$$
\left\{\begin{array} { l } 
{ R ^ { ( 0 ) } \overline { A _ { s } ^ { ( 0 ) } } = \overline { B _ { s } ^ { ( 0 ) } } R ^ { ( 0 ) } , } \\
{ R ^ { ( \infty ) } \overline { A _ { s } ^ { ( \infty ) } } = \overline { B _ { s } ^ { ( \infty ) } } R ^ { ( \infty ) } , }
\end{array} \quad \left\{\begin{array}{l}
R^{(0)} A_{u}^{(0)}=B_{u}^{(0)} R^{(0)} \\
R^{(\infty)} A_{u}^{(\infty)}=B_{u}^{(\infty)} R^{(\infty)}
\end{array}\right.\right.
$$

- Tensor structure on $\mathcal{C}^{\prime}$. The tensor product of two objects $\left(A_{1}^{(0)}, P_{1}, A_{1}^{(\infty)}\right)$ and $\left(A_{2}^{(0)}, P_{2}\right.$, $\left.A_{2}^{(\infty)}\right)$ is defined to be

$$
\left(A_{1}^{(0)}, P_{1}, A_{1}^{(\infty)}\right) \otimes\left(A_{2}^{(0)}, P_{2}, A_{2}^{(\infty)}\right)=\left(A_{1}^{(0)} \otimes A_{2}^{(0)}, P_{1} \otimes P_{2}, A_{1}^{(\infty)} \otimes A_{2}^{(\infty)}\right) .
$$

The tensor product of two morphisms $\left(R_{1}^{(0)}, R_{1}^{(\infty)}\right)$ and $\left(R_{2}^{(0)}, R_{2}^{(\infty)}\right)$ is defined componentwise, from the usual tensor product. That the tensor product of two objects is one is 
obvious; that the tensor product of two morphisms is one is not tautological, but follows from the properties of morphisms in $\mathcal{S}^{\prime}$ and $\mathcal{C}^{\prime}$.

Defining as before two fibre functors $\mathcal{C}^{\prime} \rightarrow \operatorname{Vect}_{\mathbf{C}}^{f}$ by sending $\left(A^{(0)}, P, A^{(\infty)}\right)$ to the $\mathbf{C}^{n}$ underlying $A^{(0)}$ (resp. $A^{(\infty)}$ ) and $\left(S^{(0)}, S^{(\infty)}\right)$ to $S^{(0)}$ (resp. $S^{(\infty)}$ ), one obtains again a neutral tannakian category over $\mathbf{C}$.

3.2.1.3. The equivalence of $\mathcal{E}_{f}, \mathcal{S}^{\prime}$ and $\mathcal{C}^{\prime}$. As in 3.1, two functors can be defined; first, one from solutions to equations:

$$
\left\{\begin{array}{l}
\left(A^{(0)}, M^{(0)}, A^{(\infty)}, M^{(\infty)}\right) \sim A \\
\left(F, S^{(0)}, S^{(\infty)}\right) \sim F .
\end{array}\right.
$$

Next, one from solutions to connection triples:

$$
\left\{\begin{array}{l}
\left(A^{(0)}, M^{(0)}, A^{(\infty)}, M^{(\infty)}\right) \sim\left(A^{(0)}, P, A^{(\infty)}\right), \\
\left(F, S^{(0)}, S^{(\infty)}\right) \leadsto\left(S^{(0)}, S^{(\infty)}\right) .
\end{array}\right.
$$

Both are exact $\otimes$-preserving $\mathbf{C}$-linear equivalence of categories. Note that this equivalence is compatible with the fibre functors previously introduced.

3.2.1.4. Singularities and exponents. In order to compute the connection component of the Galois groupoid we shall need to evaluate the connection matrix at various points of $\mathbf{C}^{*}$, avoiding its singularities (since $P$ is elliptic, these singularities may actually be seen as points in $\mathbf{E}_{q}$ ). For an object $\left(A^{(0)}, P, A^{(\infty)}\right)$ of $\mathcal{C}^{\prime}$ coming from an object $A$ of $\mathcal{E}_{f}$, the singularities of $P$ respectively come from:

1. $\mathcal{S}(A)$ for the meromorphic part $M=\left(M^{(\infty)}\right)^{-1} M^{(0)}$. Precisely, $\mathcal{S}(M) \subset q^{\mathbf{z}} \mathcal{S}(A)$.

2. $S p(A(0)), S p(A(\infty))$ for the semi-simple components of the $e_{q, A(-)}$ parts. Since reduction to a constant matrix is not unique, these exponents of $A$ are actually defined up to a factor in $q^{\mathbf{Z}}$.

3. $l_{q}$, the $q$-logarithm, in case $A(0)$ or $A(\infty)$ is not semi-simple.

On the other hand, we want to define $\otimes$-stable categories only, so that we have to allow for multiplication of the exponents (and the inverse, to have stability under dual taking). Thus, for $\Sigma$ a finite subset of the open set $\mathbf{C}^{*}$ and $C$ be a finitely generated subgroup of the group $\mathbf{C}^{*}$, we consider the full subcategory $\mathcal{E}_{f, \Sigma, C}$ of $\mathcal{E}_{f}$ whose objects have all singularities in $q^{\mathbf{Z}} \Sigma$ and all exponents in $q^{\mathbf{z}} C$. From the precised version of Birkhoff's lemma we draw that an equation $A$ is in $\mathcal{E}_{f, \Sigma, C}$ if and only if its exponents are in $C$ and $\mathcal{S}(M) \subset q^{\mathbf{z}_{\Sigma}}$.

Write $\bar{\Sigma}$, resp. $\bar{C}$ for the image in $\mathbf{E}_{q}$ of a finite subset $\Sigma$ of $\mathbf{C}^{*}$, resp. a finitely generated subgroup $C$ of $\mathbf{C}^{*}$. We then consider the full subcategory $\mathcal{C}_{\Sigma, C}^{\prime}$ of $\mathcal{C}^{\prime}$ whose objects are the triples $\left(A^{(0)}, P, A^{(\infty)}\right)$ such that $S p(A(0)), S p(A(\infty))$ are subsets of $q^{\mathbf{z}} C$ and $\mathcal{S}(M) \subset q^{\mathbf{z}} \Sigma$. For such objects, $\mathcal{S}(P) \subset \bar{\Sigma} \cup \bar{C}$. Moreover, $\mathcal{C}_{\Sigma, C}^{\prime}$ is a strictly full tannakian subcategory of $\mathcal{C}^{\prime}$ and it is equivalent to $\mathcal{E}_{f, \Sigma, C}$.

For an object $\mathcal{X}=\left(A^{(0)}, P, A^{(\infty)}\right)$ of $\mathcal{C}^{\prime}$, denote by $\langle\mathcal{X}\rangle$ the tannakian subcategory generated by $\mathcal{X}$. If $\mathcal{X}$ actually belongs to the subcategory $\mathcal{C}_{\Sigma, C}^{\prime}$, this entails $\langle\mathcal{X}\rangle \subset \mathcal{C}_{\Sigma, C}^{\prime}$. The object being given, the minimal choice is to take for $C$ the subgroup of $\mathbf{C}^{*}$ generated by $S p(A(0))$ and $S p(A(\infty))$ and for $\Sigma$ the singular locus of $M$. 


\subsubsection{The Galois groupoid}

Sticking to the previous definitions, we consider a groupoid $G$ with base points 0 and $\infty$ and with corresponding arrow sets $A u t^{\otimes}\left(\omega^{(0)}\right), A u t^{\otimes}\left(\omega^{(\infty)}\right), I s o^{\otimes}\left(\omega^{(0)}, \omega^{(\infty)}\right), I s o^{\otimes}\left(\omega^{(\infty)}, \omega^{(0)}\right)$.

$$
\left.A u t^{\otimes}\left(\omega^{(0)}\right) \zeta \omega^{(0)} \underset{I s o^{\otimes}\left(\omega^{(\infty)}, \omega^{(0)}\right)}{\stackrel{I s o^{\otimes}\left(\omega^{(0)}, \omega^{(\infty)}\right)}{\longleftrightarrow}} \omega^{(\infty)}\right) A u t^{\otimes}\left(\omega^{(\infty)}\right)
$$

In this section, we shall build elements of the Galois groupoid, that is, $\otimes$-automorphisms of $\omega^{(0)}$ and of $\omega^{(\infty)}$ and $\otimes$-isomorphisms from $\omega^{(0)}$ to $\omega^{(\infty)}$. We shall consistently denote by $\mathcal{X}=$ $\left(A^{(0)}, P, A^{(\infty)}\right)$ a generic object of $\mathcal{C}^{\prime}$. We then write $A^{(0)}=A_{s}^{(0)} A_{u}^{(0)}$ and $A^{(\infty)}=A_{s}^{(\infty)} A_{u}^{(\infty)}$ the Dunford decompositions.

3.2.2.1. Local automorphisms of the fibre functor. From the general facts recalled at the end of the introduction, we easily deduce the following:

1. Let $f$ be a map: $\mathbf{C}^{*} \rightarrow \mathbf{C}^{*}$. Then $\mathcal{X} \leadsto f\left(\overline{A_{s}^{(0)}}\right)$ (resp. $\mathcal{X} \leadsto f\left(\overline{A_{s}^{(\infty)}}\right)$ ) is an automorphism of $\omega^{(0)}$ (resp. of $\left.\omega^{(\infty)}\right)$. If we take $f \in \operatorname{Hom}_{\text {grp }}\left(\mathbf{C}^{*}, \mathbf{C}^{*}\right.$ ) and (by necessity) such that $f(q)=1$, we get a $\otimes$-compatible automorphism.

2. Let $\lambda \in \mathbf{C}$. Then $\mathcal{X} \leadsto\left(A_{u}^{(0)}\right)^{\lambda}$ (resp. $\mathcal{X} \leadsto\left(A_{u}^{(\infty)}\right)^{\lambda}$ ) is a $\otimes$-automorphism of $\omega^{(0)}$ (resp. of $\left.\omega^{(\infty)}\right)$.

We thus obtain subgroups $G^{(0)} \subset A u t^{\otimes}\left(\omega^{(0)}\right)$ and $G^{(\infty)} \subset A u t^{\otimes}\left(\omega^{(\infty)}\right)$. We recognize the local Galois groups found in Section 2. They are isomorphic to each other and are commutative proalgebraic groups with unipotent component $\mathbf{C}$ and semi-simple component:

$$
G_{s}^{(0)} \simeq G_{s}^{(\infty)} \simeq\left\{f \in H_{o m} m_{g r p}\left(\mathbf{C}^{*}, \mathbf{C}^{*}\right) \mid f(q)=1\right\} \simeq H_{o m}{ }_{g r p}\left(\mathbf{C}^{*} / q^{\mathbf{Z}}, \mathbf{C}^{*}\right)
$$

This is just the dual $\check{\mathbf{E}}_{q}$ of the abstract group $\mathbf{E}_{q}$. In this description, our local Galois groups are identified to a subgroup of $\mathbf{Z}^{\text {alg }}$ but they are there embedded transversally to the natural monodromy group $\mathbf{Z}$ : their intersection with the latter is the trivial subgroup.

3.2.2.2. Building elements of the connection component. We restrict here the fibre functors $\omega^{(0)}, \omega^{(\infty)}$ to some subcategory $\mathcal{C}_{\Sigma, C}^{\prime}$ (see 3.2.1.4). We put $\Sigma^{\prime}=q^{\mathbf{Z}}(\Sigma \cup C)$ and fix $a \in \mathbf{C}^{*}-\Sigma^{\prime}$. It stems tautologically from our definition of morphisms in the category $\mathcal{C}^{\prime}$ that, for any such $a, \mathcal{X} \leadsto P(a)$ is a functorial isomorphism $\omega^{(0)} \rightarrow \omega^{(\infty)}$. However, it is not, in general, a tensor isomorphism, because $P_{1}(a) \otimes P_{2}(a) \neq P_{1}(a) \underline{\otimes} P_{2}(a)$. There is of course an exception if $C$ is trivial, e.g. for regular equations. The right and left excess factors are $\Phi\left(A_{1, s}^{(0)}, A_{2, s}^{(0)}\right)$ and $\Phi\left(A_{1, s}^{(\infty)}, A_{2, s}^{(\infty)}\right)$. They can be exactly compensated by taking $e_{q, \underline{A_{s}^{(\infty)}}} P(a)\left(e_{q, A_{s}^{(0)}}\right)^{-1}$ instead of $P(a)$. However, this depends on $A_{s}^{(0)}$ and $A_{s}^{(\infty)}$ and not only on $\overline{A_{s}^{(0)}}$ and $\overline{A_{s}^{(\infty)}}$ (see the first half of the first fact in 3.2.2.1), so that it is no longer a functorial isomorphism.

In order to twist the connection matrix, one chooses, for each $a \in \mathbf{C}^{*}$, a group homomorphism $g_{a} \in H_{o m} m_{g r p}\left(\mathbf{C}^{*}, \mathbf{C}^{*}\right)$ such that $g_{a}(q)=1$. We have exhibited such a family $\left(g_{a}\right)_{a \in \mathbf{C}^{*}}$ in 2.2.3 and we shall make this choice more precise further below. One then puts, for $c \in \mathbf{C}^{*}$, 
$\psi_{a}(c)=\frac{e_{c}(a)}{g_{a}(c)}$, so that:

$$
\left\{\begin{array}{l}
\psi_{a}\left(c_{1}\right) \psi_{a}\left(c_{2}\right)=\psi_{a}\left(c_{1} c_{2}\right) \phi\left(c_{1}, c_{2}\right)(a) \\
\psi_{a}(c) \text { depends only on } \bar{c}
\end{array}\right.
$$

Now extend $\psi_{a}$ to matrices, so that:

$$
\left\{\begin{array}{l}
\psi_{a}\left(A_{1}\right) \otimes \psi_{a}\left(A_{2}\right)=\left(\psi_{a}\left(A_{1}\right) \otimes \psi_{a}\left(A_{2}\right)\right) \Phi\left(A_{1}, A_{2}\right)(a), \\
\psi_{a}(A) \text { depends only on } \bar{A}\left(\text { actually, on } \overline{A_{s}}\right) .
\end{array}\right.
$$

We have built our twisting factor. It is made up of two ingredients: one is due to the twisting of the tensor product, itself due to the noncanonical choice of solutions. The other comes from the artificial concentration of the local groupoid at 0 , in a unique base point (and a unique local group), as shown in the figure at the end of 3.2 and in Proposition 3.2.3.1 and Remark 3.2.3.2 (all the points $\omega_{a}^{(0)}$ are artificially concentrated at the unique point $\left.\omega^{(0)}\right)$. Let $a \in \mathbf{C}^{*}-\Sigma^{\prime}$. Then

$$
\mathcal{X} \leadsto \breve{P}(a)=\left(\psi_{a}\left(A_{s}^{(\infty)}\right)\right)^{-1} P(a)\left(\psi_{a}\left(A_{s}^{(0)}\right)\right)
$$

is a $\otimes$-isomorphism: $\omega^{(0)} \rightarrow \omega^{(\infty)}$. Note that choosing another group homomorphism $g_{a}$ changes it by a factor in $\check{\mathbf{E}}_{q}$ and therefore changes our twisted connection matrix $\breve{P}$ by a left and a right factor in the semi-simple component of the local Galois groups. Similarly, it does not matter that we have taken the same $g_{a}$ to twist on the 0 and on the $\infty$ side. We therefore take a slightly different choice for this family. We first write $\mathbf{C}^{*}=\mathbf{U} \times q^{\mathbf{R}}$, thereby meaning that we have chosen a logarithm of $q: q=e^{-2 \imath \pi \tau}, \operatorname{Im}(\tau)>0$. We thus write every $z \in \mathbf{C}^{*}$ : $z=u q^{y}=u e^{-2 \imath \pi \tau y}$ with $|u|=1$ and $y \in \mathbf{R}$. This allows us to define, for any $\alpha \in \mathbf{C}$, a group homomorphism:

$$
\delta_{\alpha}:\left\{\begin{array}{l}
\mathbf{C}^{*} \rightarrow \mathbf{C}^{*}, \\
u q^{y} \mapsto q^{\alpha y}=e^{-2 \imath \pi \tau \alpha y} .
\end{array}\right.
$$

To define $g_{a}$, we now choose a logarithm of $a$. We first define the function $\log _{q}$ on the whole of $\mathbf{C}^{*}$ by the following conditions: it is to be holomorphic on $\mathbf{C}^{*}-q^{\mathbf{R}}$, one has $\log _{q}\left(q^{y}\right)=y$ and the discontinuity is just before the cut when turning counterclockwise around 0 . Lastly, we put $g_{a}=\delta_{\alpha}$ where $\alpha=\log _{q}(a)$. This definition is consistent with that in 2.2.3.6, we just deal here with continuity and cuts.

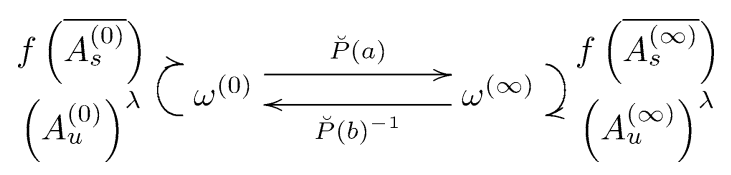

It is an important fact that $\breve{P}$ is not an elliptic function. Here are the effects of the two fundamental loops of $\pi_{1}\left(\mathbf{E}_{q}\right)$ :

- Automorphy due to the monodromy of the logarithm. Let a turn counterclockwise once around 0 . Then $g_{a}(c)=\delta_{\alpha}\left(u q^{y}\right)=e^{-2 \imath \pi \tau \alpha y}$ is multiplied by $e^{-2 \imath \pi y}$ and, since $e_{q, c}$ is uniform on $\mathbf{C}^{*}, \psi_{a}(c)$ is multiplied by $\gamma_{2}(c)$. This $\gamma_{2}$ sends $q$ to 1 , so that it defines an element $\gamma_{2}^{(0)}$ of the local Galois group at 0 (and the like at $\infty$ ): clearly, it represents the plain classical loop around 0 in $\mathbf{C}^{*}$. 
- Automorphy due to the defect of ellipticity of $\breve{P}$. From the equality $\log _{q}(q a)=\log _{q}(a)+1$, we draw: $\frac{g_{q a}}{g_{a}}=\frac{\delta_{\alpha+1}}{\delta \alpha}=\delta_{1}$, whence

$$
\frac{\psi_{q a}(c)}{\psi_{a}(c)}=\frac{e_{q, c}(q a) / g_{q a}(c)}{e_{q, c}(a) / g_{a}(c)}=\frac{c}{\delta_{1}(c)}=\gamma_{1}(c)
$$

This $\gamma_{1}$ defines again an element $\gamma_{1}^{(0)}$ of the local Galois group at 0 , similarly at $\infty$. We thus have:

$$
\breve{P}(q a)=\left(\gamma_{1}^{(\infty)}\left(A^{(\infty)}\right)\right) P(a)\left(\gamma_{1}^{(0)}\left(A^{(0)}\right)\right)^{-1} .
$$

While $\breve{P}$ is not elliptic (except in the regular case), its left and right automorphy factors under the action of $q^{\mathbf{Z}}$ are elements of the local Galois groups.

3.2.2.3. A density lemma. We again restrict ourselves to a category $\mathcal{C}_{\Sigma, C}^{\prime}$, and, occasionally, to $\mathcal{C}_{\Sigma \text {,reg. }}^{\prime}$. The arguments apply as well to the tannakian subcategory $\langle\mathcal{X}\rangle$ generated by an object $\mathcal{X}=\left(A^{(0)}, P, A^{(\infty)}\right)$. We consider a $\{0, \infty\}$-subset $E$ of the Galois groupoid $\breve{G}$, with base $\{0, \infty\}(\simeq \operatorname{Spec}(\mathbf{C} \times \mathbf{C}))$, with arrow sets $A u t^{\otimes}\left(\omega^{(0)}\right), A u t^{\otimes}\left(\omega^{(\infty)}\right), I s o^{\otimes}\left(\omega^{(0)}, \omega^{(\infty)}\right)$ and Iso ${ }^{\otimes}\left(\omega^{(\infty)}, \omega^{(0)}\right)$ with the following constraints:

- The component $E(0)$ above 0 contains the unipotent loop $\mathcal{X} \sim A_{u}^{(0)}$, and a family of semisimple loops $\mathcal{X} \leadsto \gamma_{i}\left(\overline{A_{s}^{(0)}}\right)$ where the $\gamma_{i} \in H_{\text {orm }}\left(\mathbf{C}^{*}, \mathbf{C}^{*}\right)$ are such that

$$
\bigcap_{i} \operatorname{ker} \gamma_{i}=q^{\mathbf{z}}
$$

Alternatively, if viewed as elements of $\operatorname{Hom}_{g r p}\left(\mathbf{C}^{*} / q^{\mathbf{Z}}, \mathbf{C}^{*}\right)$, the $\gamma_{i}$ are such that

$$
\bigcap_{i} \operatorname{ker} \gamma_{i}=\{1\}
$$

The component $E(\infty)$ above $\infty$ contains the corresponding elements at $\infty$.

- The component $E(0, \infty)$ above $0, \infty$ contains the paths $\mathcal{X} \sim \breve{P}(a)$ for all $a \in \mathbf{C}^{*}-\Sigma^{\prime}$. Then, the $\{0, \infty\}$-set $E$ generates a Zariski dense subgroupoid of $\breve{G}$.

\subsubsection{Relation to the results in 3.1}

We now relate the fibre functors and Galois groupoid studied here with those described previously. Let $F: A \rightarrow B$ be a morphism of flat objects, meromorphic on $\mathcal{C}^{\prime}$. Then, $F e_{q, A}=e_{q, B} S$, where $S$ has constant coefficients and $S \bar{A}=\bar{B} S$. Thus,

$$
F\left(z_{0}\right) e_{q, A}\left(z_{0}\right)=e_{q, B}\left(z_{0}\right) S
$$

that is, $\mathcal{X} \leadsto e_{q, A}\left(z_{0}\right)$ is a natural transformation from $\omega^{(0)}$ (the fibre functor in Section 3 ) to $\omega_{z_{0}}^{(0)}$. It is however not $\otimes$-compatible. On the other side, the relation $S \bar{A}=\bar{B} S$ implies that, for any map $\psi: \mathbf{C}^{*} \rightarrow \mathbf{C}^{*}$ such that $\psi(c)$ depends only on $\bar{c}, \mathcal{X} \leadsto \psi\left(\overline{A^{(0)}}\right)$ provides a natural isomorphism (not a $\otimes$-isomorphism) from $\omega^{(0)}$ to itself. Hence:

$$
\mathcal{X} \leadsto\left(\psi\left(\overline{A^{(0)}}\right)\right)^{-1} e_{q, A}\left(z_{0}\right)
$$


is again a natural transformation from $\omega^{(0)}$ to $\omega_{z_{0}}^{(0)}$. For it to be $\otimes$-preserving, it is necessary and sufficient that the map $g: c \mapsto \frac{e_{q, c}\left(z_{0}\right)}{\psi(c)}$ be a group homomorphism $\mathbf{C}^{*} \rightarrow \mathbf{C}^{*}$. Otherwise said, $\psi(c)=\frac{e_{q, c}\left(z_{0}\right)}{g(c)}$, where $g$ is a group homomorphism $\mathbf{C}^{*} \rightarrow \mathbf{C}^{*}$ and the condition $\psi(q c)=\psi(c)$ says that $g(q)=z_{0}$. We have found again the $g_{a}$ and $\psi_{a}$ of 3.2. We conclude that any such group homomorphism $g_{z_{0}}$ provides a $\otimes$-isomorphism:

$$
\mathcal{X} \leadsto g_{z_{0}}\left(\overline{A^{(0)}}\right)=g_{z_{0}}\left(\overline{A_{s}^{(0)}}\right)
$$

from $\omega^{(0)}$ to $\omega_{z_{0}}^{(0)}$. Of course, composition of such isomorphisms exactly gives the morphisms $\omega_{z_{0}}^{(0)} \rightarrow \omega_{z_{0}}^{(\infty)}$ already found.

Now, as regards the connection component, we have already seen that the connection matrix corresponding to $\left(A^{(0)}, M, A^{(\infty)}\right)$ is $P=\left(e_{q, A^{(\infty)}}\right)^{-1} M e_{q, A^{(0)}}$. Therefore, the morphism $\mathcal{X} \leadsto \breve{P}\left(z_{0}\right)$ from $\omega^{(0)}$ to $\omega^{(\infty)}$ provided by the twisted connection matrix $\breve{P}$ is but the composition:

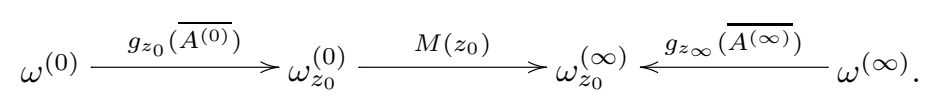

To summarize the relationship between the description of the Galois groupoid given here, in 3.2, and the previous descriptions, given in 2.2 and 3.1, we must introduce some more notations (which will not be used elsewhere). We shall call $G$ the former groupoid (its base set is the disjoint union $\mathbf{C}^{*} \amalg \mathbf{C}^{*}$ ) and $\breve{G}$ the latter one (its base set is $\{0, \infty\}$ ). Recall that $\Gamma_{a} \in I s o^{\otimes}\left(\omega_{a}^{(0)}, \omega_{a}^{(\infty)}\right)$ was defined in Proposition 3.1.2.2. The corresponding "twisted" element of $I s o^{\otimes}\left(\omega^{(0)}, \omega^{(\infty)}\right)$, defined in 3.2.2.2 as $\mathcal{X} \leadsto \breve{P}(a)$, we denote by $\breve{\Gamma}_{a}$. Similarly, for the local groupoids, we define, for $a \in \mathbf{C}^{*}$ :

$$
\begin{aligned}
& \Gamma_{a}^{(0)} \in I s o^{\otimes}\left(\omega^{(0)}, \omega_{a}^{(0)}\right) \quad \text { by } \mathcal{X} \leadsto g_{a}\left(\overline{A_{s}^{(0)}}\right) \quad \text { and } \\
& \Gamma_{a}^{(\infty)} \in I s o^{\otimes}\left(\omega_{a}^{(\infty)}, \omega^{(\infty)}\right) \quad \text { by } \mathcal{X} \leadsto g_{a}\left(\overline{A_{s}^{(\infty)}}\right) .
\end{aligned}
$$

\subsubsection{Proposition. - For all $a, \breve{\Gamma}_{a}=\Gamma_{a}^{(\infty)} \circ \Gamma_{a} \circ \Gamma_{a}^{(0)}$.}

It is moreover clear that the correspondence thus obtained between the elements of both Galois groupoids preserves the continuity of the underlying morphisms of groups, and that the fundamental groupoids are mapped to each other through this correspondance.

The following commutative diagram exhibits the relations linking elements of $G$ to elements of $\breve{G}$. The leftmost and rightmost fibre functors $\omega^{(0)}$ and $\omega^{(\infty)}$ (here evaluated on an object $\left.\mathcal{X}=\left(A^{(0)}, M, A^{(\infty)}\right)\right)$ should be thought of as base points of $\breve{G}$, while the inner vertical triangles respectively belong to the subgroupoids $G^{(0)}$ and $G^{(\infty)}$ of $G$.

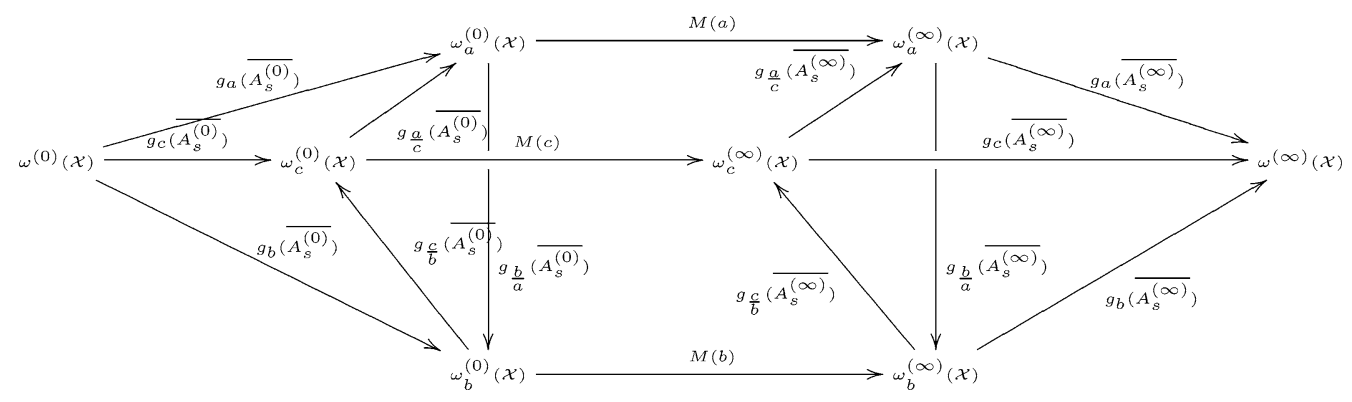


3.2.3.2. Remark. - The above diagram can also be understood as explicitly identifying $G$ with the groupoid induced by $\breve{G}$ through the canonical projection $\mathbf{C}^{*} \amalg \mathbf{C}^{*} \rightarrow\{0, \infty\}$, in the sense of [11], 1.6.

\subsection{The connection component for abelian regular equations}

In 3.2, we have given a more concrete description of the "connection component". Note however that neither description can be considered as a result of topological nature, as it requires uncountably many generating paths. A partial solution will be proposed here.

For a regular equation $A$, the Galois group at 0 is Zariski-generated by the $P(a)^{-1} P(b)$, where $a, b$ run over $\mathbf{C}^{*}-q^{\mathbf{z}} \mathcal{S}(A)$. Generally speaking, it is generated by the local component at $0, G^{(0)}$, one conjugate $\left.P \breve{P(a}\right)^{-1} G^{(\infty)} \breve{P}(a)$ of the local component at $\infty$, and the connection component, that is, the group generated by the $\breve{P}(a)^{-1} \breve{P}(b)$ where $a, b$ run over $\mathbf{C}^{*}-\Sigma^{\prime}$. In the case of torsionless equations (see 3.3.3.1), the twisting factors of $\breve{P}$ belong to the local Galois groups and one can replace the true connection component by the fake one, generated by the $P(a)^{-1} P(b)$.

We shall describe here the connection component (hence the Galois group) for regular abelian equations; by this, we mean those regular systems such that all the values of the connection matrix commute with each other, that is, such that the connection component is a commutative group.

\subsubsection{Summary of some results in [33]}

We here apply to our context results of Sections 3 and 4 of Serre's book [33], on which this section heavily relies. We identify the complex torus $\mathbf{E}_{q}=\mathbf{C}^{*} / q^{\mathbf{Z}}$ with the corresponding elliptic curve and the latter with the set of its complex points. We also identify the rational function field $k\left(\mathbf{E}_{q}\right)$ of the algebraic curve $\mathbf{E}_{q}$ with the field $\mathcal{M}\left(\mathbf{E}_{q}\right)$ of elliptic functions. The connection matrix $P$ defines a meromorphic function on $\mathbf{E}_{q}$ and we call $S$ its singular locus (made up of its poles along with those of $P^{-1}$, see 1.2.2), a finite subset of $\mathbf{E}_{q}$.

We fix once for all a base point $a_{0} \in \mathbf{E}_{q}-S$. The meromorphic mapping:

$$
\left\{\begin{array}{l}
\mathbf{E}_{q} \rightarrow G l_{n}(\mathbf{C}), \\
a \mapsto\left(P\left(a_{0}\right)\right)^{-1} P(a),
\end{array}\right.
$$

can be seen as a rational map $f: \mathbf{E}_{q} \rightarrow G l_{n}(\mathbf{C})$ and the holomorphy of $P$ on $\mathbf{E}_{q}-S$ implies that $f$ is regular on the curve $\mathbf{E}_{q}-S$. Hence the Galois group $G \subset G l_{n}(\mathbf{C})$ of $A$ is Zariski-generated by the image of the regular map $f: \mathbf{E}_{q}-S \rightarrow G l_{n}(\mathbf{C})$.

Being parameterized by a Zariski-dense subset of an irreductible projective curve, $G$ is a connected algebraic group and we have assumed it to be commutative. It is therefore the product of an algebraic torus ${ }^{5}$ and an affine space:

$$
G \simeq \mathbf{G}_{\mathbf{m}}{ }^{k} \times \mathbf{G}_{\mathbf{a}}{ }^{l}
$$

(see [6], 3.8 and 4.8 and [35], 3.4).

According to [33], Theorem 1 of Chapter 3, a module:

$$
\mathfrak{M}=\sum_{p \in S} n_{p}[p] \quad\left(\text { all } n_{p}>0\right)
$$

\footnotetext{
${ }^{5}$ To avoid any mishap, we shall systematically call complex torus an elliptic curve over $\mathbf{C}$ and algebraic torus a torus in the sense of the theory of linear algebraic groups.
} 
is associated to $f$, that is, an effective divisor on $\mathbf{E}_{q}$ with support exactly $S$. To this module is associated a generalized jacobian:

$$
\Phi_{\mathfrak{M}}: \mathbf{E}_{q} \rightarrow J_{\mathfrak{M}}
$$

where $J_{\mathfrak{M}}$ is a commutative algebraic group and $\Phi_{\mathfrak{M}}$ is a rational map defined up to a translation in $J_{\mathfrak{M}}$; for instance, fixing a base point $a_{0} \in \mathbf{E}_{q}-S$ and requiring that it be mapped to $0 \in J_{\mathfrak{M}}$ uniquely determines $\Phi_{\mathfrak{M}}$ (see Chapter 5 of [33]). We shall henceforth do so.

The generalized jacobian $J_{\mathfrak{M}}$ has the following universal property: if $\mathfrak{M}$ is associated to $f$, then $f$ has a unique factorization $f=F \circ J_{\mathfrak{M}}$ with $F: J_{\mathfrak{M}} \rightarrow G$ a (regular) morphism of algebraic groups. From the universality and the general properties of algebraic groups, it follows that $\operatorname{Im}(F)=G$.

As a matter of fact, the module $\mathfrak{M}$ is not uniquely determined by $f:$ any $\mathfrak{M}^{\prime} \geqslant \mathfrak{M}$ will do. There is then a corresponding map $J_{\mathfrak{M}^{\prime}} \rightarrow J_{\mathfrak{M}}$. Thus, we can factor $f$ through the projective limit of all $J_{\mathfrak{M}}$ with modules supported by $S$. This can be done with a fixed base point outside $S$. Call $J_{S}$ this projective limit. We shall not confuse it with the $J_{\mathfrak{M}}$ corresponding to the module $\sum_{p \in S}[p]$.

According to [33], p. 99, $J_{\mathfrak{M}}$ is an extension of the jacobian $J$ of $\mathbf{E}_{q}$ by a linear group $L_{\mathfrak{M}}$, the structure of which will be made explicit below:

$$
0 \rightarrow L_{\mathfrak{M}} \rightarrow J_{\mathfrak{M}} \rightarrow J \rightarrow 0
$$

Taking the projective limits, there is a corresponding extension:

$$
0 \rightarrow L_{S} \rightarrow J_{S} \rightarrow J \rightarrow 0
$$

In our case, $J=\mathbf{E}_{q}$. Moreover, the map $F: J_{\mathfrak{M}} \rightarrow G$ is totally determined by its restriction to $L_{\mathfrak{M}}$ : indeed, for two maps $F, F^{\prime}: J_{\mathfrak{M}} \rightarrow G$ coinciding on $L_{\mathfrak{M}}$, one would get

$$
F^{-1} F^{\prime}: J_{\mathfrak{M}} \rightarrow G
$$

trivial on $L_{\mathfrak{M}}$, thus factoring through a regular map from the projective curve $J$ to the affine group $G$, hence trivial.

To summarize, to every regular abelian object of rank $n$ with singularities in $S$, we associate a regular map from $L_{S}$ to $G l_{n}(\mathbf{C})$ the image of which is its Galois group. this correspondence is one to one and we shall hereafter make it more explicit.

\subsubsection{The abelianized of the regular fundamental group}

Still following [33], we introduce, for $p \in S$ and $n \in \mathbf{N}^{*}$, the following groups:

$$
\left\{\begin{array}{l}
U_{p}=\left\{g \in k\left(\mathbf{E}_{q}\right)^{*} \mid v_{p}(g) \geqslant 0\right\} \\
U_{p}^{(n)}=\left\{g \in k\left(\mathbf{E}_{q}\right)^{*} \mid v_{p}(1-g) \geqslant n\right\} \\
V_{p}^{(n)}=U_{p}^{(1)} / U_{p}^{(n)}
\end{array}\right.
$$

The latter is a $(n-1)$-dimensional affine space. In characteristic zero, it can be parametrized using the exponential of truncated power series, so that we can (and shall) see it as the group:

$$
V_{p}^{(n)} \simeq\left\{\exp \left(-a_{1} t-\cdots-a_{n-1} \frac{t^{n-1}}{n-1}\right)\right\}
$$


with $t$ a local parameter at $p$. This parametrization will make easier the description of morphisms to $\mathbf{G}_{\mathbf{a}}$ in 3.3.2.2. Also note that, writing $g=(g / g(0)) \times g(0)$, one has:

$$
\left\{\begin{array}{l}
U_{p} \simeq U_{p}^{(1)} \times \mathbf{G}_{\mathbf{m}}, \\
\frac{U_{p}}{U_{p}^{(n)}} \simeq V_{p}^{(n)} \times \mathbf{G}_{\mathbf{m}} .
\end{array}\right.
$$

We now define, for $\mathfrak{M}=\sum_{p \in S} n_{p}[p]\left(\right.$ all $\left.n_{p}>0\right)$ :

$$
\left\{\begin{array}{l}
R_{\mathfrak{M}}=\prod_{p \in S} \frac{U_{p}}{U_{p}^{\left(n_{p}\right)}} \simeq \mathbf{G}_{\mathbf{m}}{ }^{S} \times \prod_{p \in S} \mathbf{G}_{\mathbf{a}}{ }^{n_{p}-1} \\
\Delta=\left\{(x, \ldots, x) \in \mathbf{G}_{\mathbf{m}}{ }^{S}\right\} \quad \text { (the diagonal) } \\
L_{\mathfrak{M}}=\frac{R_{\mathfrak{M}}}{\Delta} \simeq \frac{\mathbf{G}_{\mathfrak{m}}{ }^{S}}{\Delta} \times \prod_{p \in S} \mathbf{G}_{\mathbf{a}}{ }^{n_{p}-1}
\end{array}\right.
$$

Then, going to the projective limit, we get:

$$
\left\{\begin{array}{l}
L_{S}=L_{S, s} \times L_{S, u}, \quad \text { where } \\
L_{S, s}=\frac{\mathbf{G}_{\mathbf{m}}{ }^{S}}{\Delta} \simeq \mathbf{G}_{\mathbf{m}}|S|-1 \quad \text { and } \\
L_{S, u} \simeq \prod_{p \in S}\left(1+t_{p} \mathbf{C} \llbracket t_{p} \rrbracket\right) .
\end{array}\right.
$$

The groups $L_{S, s}$ and $L_{S, u}$ are respectively the semisimple and the unipotent factor of the Jordan decomposition of the commutative algebraic group $L_{S}$ (see [6], I.4.5). Here, for each $p \in S$ we have, selected a local parameter $t_{p}$ at $p$ and identified the projective limit of the $\mathbf{G}_{\mathbf{a}}{ }^{n_{p}-1}$ to

$$
1+t_{p} \mathbf{C} \llbracket t_{p} \rrbracket=\exp \left\{-a_{1} t-\cdots-a_{n-1} \frac{t^{n-1}}{n-1}-\cdots\right\}
$$

To all our regular abelian objects of order $n$ with singular locus on $S$, we have associated injectively a regular morphism of algebraic groups from $L_{S}$ to $G l_{n}(\mathbf{C})$. To find precisely our candidate for the abelianized regular fundamental group with singularities in $S$ :

$$
\pi_{a b, S, \mathrm{reg}}^{1}=\frac{\pi_{S, \mathrm{reg}}^{1}}{\left[\pi_{S, \mathrm{reg}}^{1}, \pi_{S, \mathrm{reg}}^{1}\right]},
$$

we have to check which morphisms: $L_{S} \rightarrow G l_{n}(\mathbf{C})$ actually arise from abelian objects in $\mathcal{C}_{\Sigma \text {,reg }}$. Dealing with commutative groups, we just have to find all maps to $\mathbf{G}_{\mathbf{m}}$ and to $\mathbf{G}_{\mathbf{a}}$, that is, 1-dimensional and unipotent 2-dimensional objects. The following (again) comes from [33] (paragraph 18 and the description of local symbols in Chapter 3).

3.3.2.1. The semi-simple component. Let $f: \mathbf{E}_{q} \rightarrow \mathbf{G}_{\mathbf{m}}$, which we identify with an elliptic function with poles and zeros in $S$. The corresponding map on $L_{S, u}$ is trivial. On the $p$ component $(p \in S)$ of $L_{S, s}$, it is given by $x \mapsto x^{v_{p}(f)}$, the triviality on the diagonal $\Delta$ being forced by the residue formula: $\sum_{p \in S} v_{p}(f)=0$.

Such an elliptic function is characterized, up to a factor in $\mathbf{C}^{*}$, by its divisor $\sum v_{p}(f)[p]$. The latter is bound by the following conditions:

$$
\left\{\begin{array}{l}
\sum_{p \in S} v_{p}(f)=0, \\
\sum_{p \in S} v_{p}(f) p=0_{\mathbf{E}_{q}}
\end{array}\right.
$$


Conversely, these conditions characterize the divisors of elliptic functions. We want to get rid of all elements of $L_{S, s}$ that are killed by such divisors. Therefore, we put:

$$
\left\{\begin{array}{l}
\operatorname{Rel}_{\mathbf{E}_{q}}(S)=\left\{\left(n_{p}\right)_{p \in S} \in \mathbf{Z}^{S} \mid \sum_{p \in S} n_{p} p=0_{\mathbf{E}_{q}}\right\}, \\
L_{S, s}^{\prime}=\text { image in } L_{S, s} \text { of }\left\{\left(x_{p}\right)_{p \in S} \mid \forall\left(n_{p}\right)_{p \in S} \in \operatorname{Rel}_{\mathbf{E}_{q}}(S), \prod_{p \in S} x_{p}^{n_{p}}=1\right\} .
\end{array}\right.
$$

And we can now put:

$$
\pi_{a b, S, \mathrm{reg}, s}^{1}=\frac{L_{S, s}}{L_{S, s}^{\prime}} .
$$

3.3.2.2. The unipotent component. We make it explicit by considering unipotent rank 2 objects: $\left(\begin{array}{ll}1 & f \\ 0 & 1\end{array}\right)$, where $f: \mathbf{E}_{q} \rightarrow \mathbf{G}_{\mathbf{a}}$ is rational with all poles on $S$ (zeros do not matter here).

The corresponding effect on $L_{S, s}$ is trivial. The effect on $L_{S, u}$ is trivial only at $p$-components such that $p$ is a pole: $v_{p}(f)=-k, k>0$. Then, using our previous "logarithmic" parametrization, it is given by: $\left(a_{n}\right)_{n \geqslant 1} \mapsto a_{k}$. Since we can prescribe arbitrarily the orders of the poles of $f$ just by putting zeros elsewhere, we get the whole dual of $L_{s, u}$ and may conclude:

$$
\pi_{a b, S, \mathrm{reg}, u}^{1}=L_{S, u} .
$$

3.3.2.3. THEOREM. - The abelian regular objects with singularities in $S$ are classified by the representations of the following algebraic group (see Eq. (3)):

$$
\pi_{a b, S, \mathrm{reg}}^{1}=\frac{L_{S, s}}{L_{S, s}^{\prime}} \times L_{S, u} .
$$

This group can be seen as the abelianized regular fundamental group with singular locus carried by $S$.

3.3.2.4. Example (dimension 1). - Here is an explicit computation in dimension 1 . We consider the equation $\sigma_{q} y=a y$, where:

$$
a(z)=a_{0} \prod_{i=1}^{r} \frac{1-u_{i}^{-1} z}{1-v_{i}^{-1} z}=a_{\infty} \prod_{i=1}^{r} \frac{1-u_{i} w}{1-v_{i} w} .
$$

One has used $w=1 / z$; the above requires that $a_{\infty} \prod u_{i}=a_{0} \prod v_{i}$. Then the connection number:

$$
p(z)=\frac{e_{q, a_{0}}(z)}{e_{q, a_{\infty}^{-1}}(w)} \prod_{i=1}^{r} \frac{u_{i} \Theta_{q}\left(z / u_{i}\right)}{v_{i} \Theta_{q}\left(z / v_{i}\right)}
$$

is elliptic. In the regular case, one has $a_{0}=a_{\infty}=1, \prod u_{i}=\prod v_{i}$ and the connection number is:

$$
p(z)=\prod_{i=1}^{r} \frac{u_{i} \Theta_{q}\left(z / u_{i}\right)}{v_{i} \Theta_{q}\left(z / v_{i}\right)} .
$$

The connection component is the subgroup of $\mathbf{C}^{*}$ generated by the values $\frac{p(b)}{p(a)}$, where $a, b$ run through $\mathbf{C}^{*}-\left\{u_{1}, \ldots, u_{r}, v_{1}, \ldots, v_{r}\right\}$. One can of course fix $a$. This group is clearly connected, so it has to be $\mathbf{C}^{*}$ (the general case) or trivial. The latter occurs if $p(z)$ is constant, that is, if the given equation is (equivalent to) the trivial equation $\sigma_{q} f=f$. 
3.3.2.5. Example (dimension 2, unipotent connection component). - One considers the system:

$$
A(z)=\left(\begin{array}{cc}
1 & a(z) \\
0 & 1
\end{array}\right),
$$

where $a(z) \in \mathbf{C}(z)$ is such that $a(0)=a(\infty)=0$. Then the connection matrix is:

$$
P(z)=\left(\begin{array}{cc}
1 & p(z) \\
0 & 1
\end{array}\right)
$$

where $p(z)=\sum_{n \in \mathbf{Z}} a\left(q^{n} z\right)$. The connection component is:

$$
\left\{\left(\begin{array}{cc}
1 & \alpha \\
0 & 1
\end{array}\right) \mid \alpha \in G\right\}
$$

where $G$ is the subgroup of $\mathbf{C}$ generated by the $p(a)-p(b)$. It is generally equal to $\mathbf{C}$ and so is the connection component. The only exceptional case is when $p$ is constant, which means that $A$ is rationally equivalent to the trivial equation $\sigma_{q} X=X$.

3.3.2.6. Remark (torsionless equations). - We consider the case of equations with torsionfree local components. Various conditions on the exponents deserve that name; we shall require that the set of exponents at 0 (resp. at $\infty$ ) modulo $q^{\mathbf{Z}}$ be a free subset of $\mathbf{E}_{q}$. Then, for each map $g: \mathbf{C}^{*} / q^{\mathbf{Z}} \rightarrow \mathbf{C}^{*}$, one can find a group homomorphism $f: \mathbf{C}^{*} / q^{\mathbf{Z}} \rightarrow \mathbf{C}^{*}$ such that $g\left(A_{s}^{(0)}\right)=f\left(\overline{A_{s}^{(0)}}\right)$, so that the former belongs to $G^{(0)}$; similarly at $\infty$. This implies that the twisting factors in each particular $\breve{P}(a)$ belong to the local Galois groups, so that the values $P(a)$ of the untwisted connection matrix belong to the Galois groupoid, and moreover generate it along with the local groups. So we can replace the true connection component by the fake connection component generated by the $P(a)^{-1} P(b)$. To the latter, the content of 3.3.2 applies word for word. For more details, see [26], second part, 3.1.2.2.

\section{Additional results}

\subsection{Confluence of galoisian automorphisms}

This paragraph extends to the Galois group (more precisely, to the fundamental groupoid as we have defined it) the confluence results obtained in [27]. These results are closely related to semicontinuity results obtained by Yves André, see [1], in an algebro-geometric setting. Our results are less general but more explicit, since we follow specified elements along $q$-paths in $\mathbf{C}^{*}$. However, these results are not very complete since we do not know the whole story about relations.

According to the general assumptions in [27], Chapters 3 and 4, we shall consider the matrix $A$ of a fuchsian $q$-difference equation, depending on $q$ in such a way that:

$$
\frac{A-I_{n}}{q-1} \rightarrow \tilde{B}
$$

where the differential equation is fuchsian and non resonant at 0 and $\infty$, which entails that the $q$-difference equation also is for $q$ close enough to 1 . Calling $\tilde{z}_{1}, \ldots, \tilde{z}_{r}$, we assume the convergence to be uniform on any compact subset of $\mathbf{C}^{*}-\bigcup_{1 \leqslant j \leqslant r} \tilde{z}_{j} q_{0}^{\mathbf{R}}$. Last, we assume the Jordan structures at 0 and $\infty$ to vary "flatly" (see loc. cit. for a precise formulation). 
We shall then attach to $A$ the canonical triple $(A(0), P, A(\infty))$ as defined in loc. cit. in the non resonant case. Of course, we shall have this triple vary along with $\varepsilon$ and $q$.

\subsubsection{General conventions}

First, we shall slightly modify the choice of $q$-characters and $q$-logarithm, so as to get simpler determinations of their limits as $q \rightarrow 1$. We take:

$$
\left\{\begin{array}{l}
e_{q, c}(z)=z^{\varepsilon(c)} \frac{\Theta_{q}(-z)}{\Theta_{q, \bar{c}}(-z)}, \\
l_{q}(z)=-z \frac{\Theta_{q}^{\prime}(-z)}{\Theta_{q}(-z)} .
\end{array}\right.
$$

Following the conventions of [27], we have $q$ tend to 1 along a fixed logarithmic spiral. We fix $q_{0}=e^{-2 \imath \pi \tau_{0}}$, with $\operatorname{Im}\left(\tau_{0}\right)>0$ and take $q=q_{0}^{\varepsilon}=e^{-2 \imath \pi \tau}$, where $\tau=\tau_{0} \varepsilon, \varepsilon>0$. We shall have $\varepsilon$ tend to 0 along $\mathbf{R}_{+}^{*}$. The following assertions are proven in loc. cit.:

1. For $\varepsilon \rightarrow 0^{+}$, let $c_{\varepsilon} \in \mathbf{C}^{*}$ be such that $\frac{c_{\varepsilon}-1}{q-1} \rightarrow \gamma \in \mathbf{C}$. Then $e_{q, c_{\varepsilon}}(z) \rightarrow z^{\gamma}$.

2. In the same circumstances, $(q-1) l_{q}(z) \rightarrow \log z$.

Here, we take $\log z=2 \imath \pi x$ and $z^{\gamma}=e^{2 \imath \pi \gamma x}$, where we have written $z=e^{2 \imath \pi x}, x=u+v \tau_{0}$, $u \in]-\frac{1}{2}, \frac{1}{2}\left[\right.$. Said otherwise, we have taken a cut along $-q_{0}^{\mathbf{R}}$.

Then, we must choose loops in the local components. We start with the "fundamental level" value $q=q_{0}, \varepsilon=1$; afterwise, we shall need a calibration (or "renormalisation") to handle the "level $\varepsilon$ ". We split:

$$
\mathbf{C}=\mathbf{R} \oplus \mathbf{R} \tau_{0}
$$

and, writing $x=u+v \tau_{0}, u, v \in \mathbf{R}$, we define:

$$
\left\{\begin{array}{l}
x \stackrel{p_{1}}{\longmapsto} u, \\
x \stackrel{\lambda p_{2}}{\longmapsto} \lambda v .
\end{array}\right.
$$

These are group homomorphisms $\mathbf{C} \rightarrow \mathbf{R}$ sending $\mathbf{Z}$ to $\mathbf{Z}$, thereby defining:

$$
\left\{\begin{array}{l}
z \stackrel{\gamma_{1}}{\longmapsto} e^{2 \imath \pi u} \\
z \stackrel{\gamma_{2}^{\lambda}}{\longmapsto} e^{2 \imath \pi \lambda v}
\end{array}\right.
$$

(we have written $z=e^{2 \imath \pi x}$ ). These are group homomorphisms: $\mathbf{C}^{*} \rightarrow \mathbf{C}^{*}$, with images in the unit circle $\mathbf{U}$.

We want to relate these loops ${ }^{6}$ to our previous fundamental loops in the semi-simple local components at level $q=q_{0}^{\varepsilon}$ :

$$
G_{q}^{(0)}=H_{o m}{ }_{g r p}\left(\mathbf{C}^{*} / q^{\mathbf{Z}}, \mathbf{C}^{*}\right) .
$$

We see, writing $x=u+v \tau_{0}=u+\frac{v}{\varepsilon} \tau$ that the latter are precisely $\gamma_{1}$ and $\gamma_{2}^{\varepsilon}$.

In the same way, we have to "renormalize" the twisting factors in $\breve{P}$, mainly the $g_{a}$. This is done writing, as before, $a=e^{2 \imath \pi \alpha}$ and taking as $g_{a}$ the group homomorphism from $\mathbf{C}^{*}$ to $\mathbf{C}^{*}$ induced by

(the latter clearly sends $\mathbf{Z}$ to $\mathbf{Z}$ ).

$$
\left\{\begin{array}{l}
\mathbf{C} \rightarrow \mathbf{C}, \\
u+v \tau_{0} \mapsto-\frac{v}{\varepsilon} \alpha
\end{array}\right.
$$

\footnotetext{
${ }^{6}$ We shall concentrate on the component at 0 , the case of $\infty$ being obviously the same.
} 
We must now choose galoisian automorphisms at the level $\varepsilon=0$, that is, for the limit differential equation. The latter has a pole at 0 , so that its local Galois group is an image in $G L_{n}(\mathbf{C})$ of $\pi_{1}\left(\left(\mathbf{C}^{*}, 0\right), .\right)^{\text {alg }}=\mathbf{Z}^{\text {alg }}$. We only take care here of the semi-simple component $\mathbf{Z}_{s}^{\text {alg }}=H_{\text {orm }}\left(\mathbf{C}^{*}, \mathbf{C}^{*}\right)$. Unhappily, we shall not arrive at the usual fundamental loop $1 \in \mathbf{Z}$, here identified with $I d_{\mathbf{C}^{*}} \in H_{o m} \operatorname{mrp}\left(\mathbf{C}^{*}, \mathbf{C}^{*}\right)$. To define specific elements, we split:

$$
\mathbf{C}=\frac{1}{\tau_{0}} \mathbf{R} \oplus \mathbf{R}
$$

and, writing $x^{\prime}=\frac{u^{\prime}}{\tau_{0}}+v^{\prime}, u^{\prime}, v^{\prime} \in \mathbf{R}$, we define:

$$
\left\{\begin{array}{l}
x^{\prime} \stackrel{w \tilde{p}_{1}}{\longmapsto} w u^{\prime} \\
x^{\prime} \stackrel{\tilde{p}_{2}}{\longmapsto} v^{\prime}
\end{array}\right.
$$

These group homomorphisms $\mathbf{C} \rightarrow \mathbf{C}$ send $\mathbf{Z}$ to $\mathbf{Z}$ and, writing $z^{\prime}=e^{2 \imath \pi x^{\prime}}$, we can define:

$$
\left\{\begin{array}{l}
z^{\prime} \stackrel{\tilde{\gamma}_{1}^{w}}{\longmapsto} e^{2 \imath \pi w u^{\prime}} \\
z^{\prime} \stackrel{\tilde{\gamma}_{2}}{\longmapsto} e^{2 \imath \pi v^{\prime}} .
\end{array}\right.
$$

These loops at 0 define elements of the semi-simple component of the local Galois group of the equation

$$
\delta \tilde{X} \stackrel{\text { def }}{=} z \frac{d}{d z} \tilde{X}=\tilde{B} \tilde{X}
$$

through the matrices $\gamma_{i}\left(e^{2 \imath \pi \tilde{B}(0)}\right)$. Here, the differential equation is assumed to be fuchsian at 0 , so that $\tilde{B}(0) \in M_{n}(\mathbf{C})$. These matrices generate a Zariski-dense subgroup of the local Galois group, though not the monodromy group: the latter is generated by $e^{2 \imath \pi \tilde{B}(0)}$, which comes from $\tilde{\gamma}_{1}^{1 / \tau_{0}} \tilde{\gamma}_{2}$.

\subsubsection{Confluence of the connection component}

From [27], we know that $P$ tends to $\tilde{P}$, a matrix that is locally constant on the nonconnected open subset

$$
\tilde{\Omega}=\mathbf{C}^{*}-\bigcup_{0 \leqslant j \leqslant r} \tilde{z}_{j} q_{0}^{\mathbf{R}}
$$

of $\mathbf{S}$, where we have put, for simplicity, $\tilde{z}_{0}=1$. Of course, $q_{0}^{\mathbf{R}}=e^{-2 \imath \pi \tau_{0} \mathbf{R}}$.

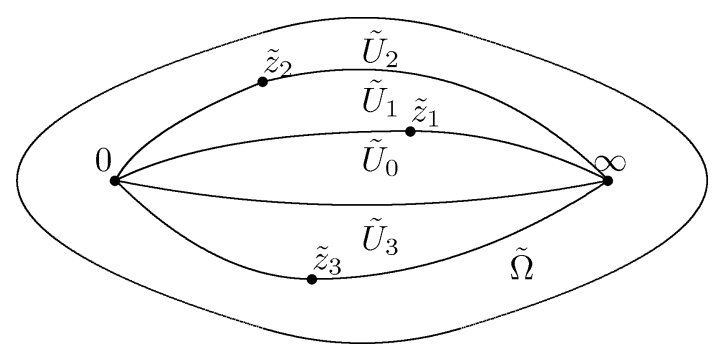

The boundary of $\tilde{\Omega}$ is made up of the $q$-spirals generated by 1 and the singularities $\tilde{z}_{i}$ of $\tilde{B}$. 
The matrix $\tilde{P}$ takes a finite number of values $\tilde{P}\left(a_{i}\right), 0 \leqslant i \leqslant r$, and the $\left(\tilde{P}\left(a_{i}\right)\right)^{-1} \tilde{P}\left(a_{i-1}\right)$, $1 \leqslant i \leqslant r$, are the monodromy operators at singularities other than $0, \infty$. But we have built our Galois isomorphisms with $\breve{P}$ instead of $P$, so we have to study the fate of $\psi_{a}\left(A_{s}^{(0)}\right)$ and $\psi_{a}\left(A_{s}^{(\infty)}\right)$ as $q \rightarrow 1$.

Under the confluence assumptions, the exponents $c$ of $A$ at 0 , resp. at $\infty$ are such that $\frac{c-1}{q-1} \rightarrow \tilde{c}$, the exponents of $\tilde{B}$ at 0 , resp. at $\infty$. We shall do the computation with $c=q^{\tilde{c}}$, which, according to the lemma of [27], 3.1, does not matter. Following 4.1.1, we find that $e_{q, c}(a) \rightarrow e^{2 \imath \pi \tilde{c} a}$ then $g_{a}(c) \rightarrow e^{2 \imath \pi v^{\prime}}$, where we have written $\tilde{c}=\frac{u^{\prime}}{\tau_{0}}+v^{\prime}, u^{\prime}, v^{\prime} \in \mathbf{R}$. Last, we obtain $\psi_{a}(c) \rightarrow e^{2 \imath \pi \alpha u^{\prime} / \tau_{0}}$ : the twisting factor tends to $\tilde{\gamma}_{1}^{\alpha / \tau_{0}}$. To be precise:

$$
\breve{P}(a) \rightarrow \tilde{\gamma}_{1}^{\frac{\alpha}{\tau_{0}}}\left(e^{2 \imath \pi \tilde{B}(\infty)}\right) \tilde{P}(a)\left(\tilde{\gamma}_{1}^{\frac{\alpha}{\tau_{0}}}\left(e^{2 \imath \pi \tilde{B}(0)}\right)\right)^{-1} .
$$

Therefore, up to factors from the local Galois groups at 0 and $\infty$, we get the whole system of monodromy factors at other singularities.

\subsubsection{Confluence of the local components}

4.1.3.1. Unipotent part. The unipotent loop at 0 defined at the beginning of 2.2 .3 gives rise to a continuous family of Galois automorphisms $A_{u}^{(0)}$. We renormalize and follow instead the $\left(A_{u}^{(0)}\right)^{-1 / \tau}$ : the limit is plainly the unipotent Galois automorphism at level $0, e^{2 \imath \pi \tilde{B}_{n}(0)}$, obtained from the nilpotent component in the additive Dunford decomposition of $\tilde{B}(0)$.

4.1.3.2. Semi-simple part: generators. As noticed before, the exponents $c$ of $A$ at 0 are such that $\frac{c-1}{q-1} \rightarrow \tilde{c}$, the exponents of $\tilde{B}$ at 0 . Again, we compute with the innocuous assumption that $c=q^{\tilde{c}}$ and we write $\tilde{c}=\frac{u^{\prime}}{\tau_{0}}+v^{\prime}, u^{\prime}, v^{\prime} \in \mathbf{R}$. Then:

$$
\left\{\begin{array}{l}
\gamma_{1}(c)=e^{2 \imath \pi u^{\prime} \varepsilon} \rightarrow 1, \\
\gamma_{2}(c)=e^{2 \imath \pi v^{\prime}} \rightarrow \tilde{\gamma}_{2}(\tilde{c}) .
\end{array}\right.
$$

We obtain eventually:

$$
\left\{\begin{array}{l}
\gamma_{1}\left(A_{s}^{(0)}\right) \rightarrow I_{n}, \\
\gamma_{2}\left(A_{s}^{(0)}\right)=\tilde{\gamma}_{2}\left(e^{2 \imath \pi \tilde{B}(0)}\right) .
\end{array}\right.
$$

The loop $\gamma_{2}$ (which we interpreted as the plain loop around 0 in $\mathbf{C}^{*}$ ) turns infinitely fast, thus compensating the trivialization of the exponents (which tend to 1). The loop $\gamma_{1}$ (which we interpreted as the start of the move to infinity) turns at constant speed, so that we must accelerate it to compensate for the trivialization. We therefore consider $\gamma_{1}^{E(1 / \varepsilon)}$ and find that this will do:

$$
\gamma_{1}\left(A_{s}^{(0)}\right)^{[1 / \varepsilon]} \rightarrow \tilde{\gamma}_{1}\left(e^{2 \imath \pi \tilde{B}(0)}\right)
$$

The trip is not so smooth, involving jumps at $1 / m, m \in \mathbf{N}^{*}$. In the end, we have reached the whole subgroup generated by $\tilde{\gamma}_{1}$ and $\tilde{\gamma}_{2}$.

4.1.3.3. Semi-simple part: relations. Again, we consider exponents that vary along $q$ spirals: $q^{\gamma_{1}}, \ldots, q^{\gamma_{n}}$. One must compare the multiplicative relations of the $q^{\gamma_{i}}$ with the additive relations of the $\gamma_{i}$ modulo $\mathbf{Z}$. We therefore introduce the module of relations:

$$
L=\left\{\left(m_{1}, \ldots, m_{n}\right) \in \mathbf{Z}^{n} \mid m_{1} \gamma_{1}+\cdots+m_{n} \gamma_{n} \in \mathbf{Z}\right\} .
$$


Writing $\gamma_{i}=\frac{a_{i}}{\tau_{0}}+b_{i}$, with $a_{i}, b_{i} \in \mathbf{R}$, the above condition is equivalent to:

$$
\left\{\begin{array}{l}
m_{1} a_{1}+\cdots+m_{n} a_{n}=0 \\
m_{1} b_{1}+\cdots+m_{n} b_{n} \in \mathbf{Z}
\end{array}\right.
$$

Now define the "exceptional set":

$$
E=\left\{\varepsilon>0 \mid 1 / \varepsilon \in \mathbf{Q} a_{1}+\cdots+\mathbf{Q} a_{n}\right\} .
$$

This is an enumerable set and it is clear that, for $\varepsilon \notin E$, the local $q$-difference Galois group at level $\varepsilon$ has no more relations than the local differential Galois group at level 0.

\subsubsection{Description of the monodromy action with a fixed base point}

Consider a fixed base point $a_{0} \in \tilde{U}_{0}$ such that $\left|a_{0}\right|<\left|\tilde{z}_{i}\right|$ for $0 \leqslant i \leqslant r$. In each slice $\tilde{U}_{i}$, choose $a_{i}$ such that $\left|a_{i}\right|=\left|a_{0}\right|$. Then, for $i=1, \ldots, r$, we can define a loop with base point $a_{0}$ in the following way: it goes from $a_{0}$ to $a_{i-1}$ along a simple circle arc with center 0 , counterclockwise; it turns once counterclockwise around $\tilde{z}_{i}$, crossing $q_{0}^{\mathbf{R}} \tilde{z}_{i}$ exactly twice; it comes back from $a_{i-1}$ to $a_{0}$ through the same circle arc. Thus, we get well defined elements:

$$
\Gamma_{i} \in \pi_{1}\left(\mathbf{C}^{*}-\left\{\tilde{z}_{1}, \ldots, \tilde{z}_{r}\right\} ; a_{0}\right), \quad i=1, \ldots, r .
$$

Together with $\Gamma_{0}$, the class of the simple positive circle around 0 , they form a family of free generators of $\pi_{1}\left(\mathbf{C}^{*}-\left\{\tilde{z}_{1}, \ldots, \tilde{z}_{r}\right\} ; a_{0}\right)$.

The monodromy action of $\Gamma_{0}$ on the space of solutions of the differential equation $\delta \tilde{X}=\tilde{B} \tilde{X}$ as well as the action of the simple loop around $\infty,\left(\Gamma_{0} \Gamma_{1} \cdots \Gamma_{r}\right)^{-1}$, are obtained by confluence of the local $q$-difference Galois groups, as seen in 4.1.3. To be precise, only differential galoisian automorphisms were reached this way, but with the same Zariski closures as these fundamental loops.

The monodromy action of $\Gamma_{i}$ for $1 \leqslant i \leqslant r$ has matrix:

$$
\left(\tilde{P}\left(a_{i}\right)\right)^{-1} \tilde{P}\left(a_{i-1}\right)=\lim _{q \rightarrow 1}\left(P\left(a_{i}\right)\right)^{-1} P\left(a_{i-1}\right) .
$$

To compare it with the galoisian automorphisms $\left(\breve{P}\left(a_{i}\right)\right)^{-1} \breve{P}\left(a_{i-1}\right)$, one just has to insert the twisting factors shown in 4.1.2.

\subsection{Extension to the $p$-adic case}

We indicate here briefly how most of the previous results can be extended to the context of $p$ adic $q$-difference equations. The possibility to do this rests on Tate's theory of the uniformization of rigid elliptic curves; it was suggested by Yves André ${ }^{7}$. More details are to be found in $[26,31]$, along with detailed references to the literature.

\subsubsection{Classification}

We take as a base field the completion $\mathbf{C}_{p}$ of the algebraic closure $\overline{\mathbf{Q}_{p}}$ of the field $\mathbf{Q}_{p}$ of $p$-adic numbers. It is an algebraically closed complete non archimedian valued field. One can define, for $q \in \mathbf{C}_{p}^{*}$ such that $|q|<1$, an analytic curve $\mathbf{E}_{q}=\mathbf{C}_{p}^{*} / q^{\mathbf{Z}}$ whose meromorphic function field $\mathcal{M}\left(\mathbf{E}_{q}\right)$ is an elliptic field (i.e. algebraic function field of genus 1, see [8]), so that $\mathbf{E}_{q}$ can be

\footnotetext{
${ }^{7}$ Marius van der Put told us that the results in [20] could be similarly extended.
} 
identified to an elliptic curve over $\mathbf{C}_{p}$. One gets in this way exactly those elliptic curves whose modular invariant $j\left(\mathbf{E}_{q}\right)$ is not an integer. The uniformization of such an elliptic curve is obtained with the help of the $p$-adic theta function:

$$
\Theta(z)=\prod_{n \geqslant 0}\left(1-q^{n} z\right) \prod_{n \geqslant 1}\left(1-q^{n} z^{-1}\right)
$$

This has all the properties we used to define our fundamental solutions of constant coefficient systems. Hence our abelian $\mathbf{C}_{p}$-linear rigid tensor categories $\mathcal{E}_{f}, \mathcal{S}$ and $\mathcal{C}$ can be defined, as well as the exact $\mathbf{C}_{p}$-linear $\otimes$-functors $S E$ and $S C$, our equivalence theorems remain valid here, as well as the choice for the fibre functors.

\subsubsection{The connection component}

To build as in 3.2 the matrix $\breve{P}$, we needed a morphism $g_{a}: \mathbf{C}_{p}^{*} \rightarrow \mathbf{C}_{p}^{*}$ sending $q$ to $a$. Here, for lack of an exponential, we shall resort to a more Zornian construction.

4.2.2.1. LEMMA. - Let $K$ be an algebraically closed field of characteristic 0 and let $x \in$ $K^{*}-\mu_{\infty}\left(K^{*}\right)$, where $\mu_{\infty}\left(K^{*}\right)$ is the torsion subgroup (roots of unity) of $K^{*}$. Then $K^{*}$ and $K^{*} / x^{\mathbf{Z}}$ are respectively isomorphic to $(\mathbf{Q} / \mathbf{Z}) \times \mathbf{Q} \times V$ and to $(\mathbf{Q} / \mathbf{Z}) \times(\mathbf{Q} / \mathbf{Z}) \times V$, where $V$ is a $\mathbf{Q}$-vector space and where $x \in K^{*}$ corresponds to the element $(\overline{0}, 1,0)$ of $(\mathbf{Q} / \mathbf{Z}) \times \mathbf{Q} \times V$.

This lemma guarantees the existence of the group homomorphisms $g_{a}$. Thus, our construction of $\breve{P}$ and our density lemma in 3.2 remain valid.

\subsubsection{The local components}

We now make more precise our choice of the $g_{a}$. Let $a \in \mathbf{C}_{p}^{*}$ correspond to $(\bar{\alpha}, \beta, \xi) \in$ $(\mathbf{Q} / \mathbf{Z}) \times \mathbf{Q} \times V$ and choose a lifting (a logarithm!) $\alpha$ of $\bar{\alpha}$ in $\mathbf{Q}$. Then the morphism:

$$
\left\{\begin{array}{l}
(\mathbf{Q} / \mathbf{Z}) \times \mathbf{Q} \times V \rightarrow(\mathbf{Q} / \mathbf{Z}) \times \mathbf{Q} \times V, \\
\left(\overline{\alpha^{\prime}}, \beta^{\prime}, \xi^{\prime}\right) \mapsto\left(\overline{\beta^{\prime} \alpha}, \beta^{\prime} \beta, \beta^{\prime} \xi\right)
\end{array}\right.
$$

is well defined and corresponds to a group homomorphism $\mathbf{C}_{p}^{*} \rightarrow \mathbf{C}_{p}^{*}$ sending $q$ to $a$. We now obtain naturally our fundamental semi-simple loops $\gamma_{1}$ and $\gamma_{2}$ :

1. If we change the "logarithm" $\alpha$ to $\alpha+1, g_{a}$ is changed to $g_{a}^{\prime}$ in such a way that $g_{a}^{\prime} / g_{a}$ corresponds to:

$$
\left(\overline{\alpha^{\prime}}, \beta^{\prime}, \xi^{\prime}\right) \mapsto\left(\overline{\beta^{\prime}}, 0,0\right) .
$$

This morphism we take as $\gamma_{1}: \mathbf{C}_{p}^{*} \rightarrow \mathbf{C}_{p}^{*}$. It does send $q$ to 1 .

2. If we compute $\psi_{q a} / \psi_{a}$, we get $c \mapsto \frac{c}{g_{q a}(c) / g_{a}(c)}$, corresponding to:

$$
\left(\overline{\alpha^{\prime}}, \beta^{\prime}, \xi^{\prime}\right) \mapsto\left(\overline{\alpha^{\prime}}, 0, \xi^{\prime}\right) .
$$

This morphism we take as $\gamma_{2}: \mathbf{C}_{p}^{*} \rightarrow \mathbf{C}_{p}^{*}$. It also sends $q$ to 1 .

It is obvious from the description with $\left(\overline{\alpha^{\prime}}, \beta^{\prime}, \xi^{\prime}\right)$ that $\operatorname{Ker} \gamma_{1} \cap \operatorname{Ker} \gamma_{2}=q^{\mathbf{Z}}$. Thus, our description of the local monodromy groups in 3.2 is still valid here almost naturally, that is, up to the choice of a logarithm.

\section{Acknowledgements}

Part of the present work constitutes the second part of my thesis, that was prepared under the direction of Jean-Pierre Ramis. But the whole investigation has been deeply influenced by 
his vision. I thank him heartily for his precious support. I have also been greatly helped by suggestions of the referees of that thesis, Yves André and Marius van der Put. I am grateful for many fruitful discussions with Bertrand Toen, Joseph Tapia, Lucia Di Vizio and Changgui Zhang. Last, but not least, I owe thanks to the referee of this paper, whose outstanding patience allowed to increase dramatically the precision and understandability of the text.

A first version of this work was circulated in autumn 2000, with substantially the same contents. Then, in june 2001, Bernard Malgrange gave me a copy of the paper [3] of 1996, by Baranovsky and Ginzburg, emphasizing the use of vector bundles over an elliptic curve to classify $q$-difference systems. The point of view is strikingly similar, there are however important differences (see Section 2.3.4).

\section{REFERENCES}

[1] ANDRÉ Y., Différentielles non-commutatives et théorie de Galois différentielle ou aux différences, Ann. Scient. Éc. Norm. Sup. 34 (2001) 685-739.

[2] ARnold V.I., Ordinary Differential Equations, in: Dynamical Systems, Encyclopaedia of Mathematical Sciences, vol. 1, Springer-Verlag, 1980.

[3] Baranovsky V., Ginzburg V., Conjugacy Classes in Loop Groups and G-Bundles on Elliptic Curves, in: International Mathematics Research Notes, vol. 15, 1996.

[4] Bertrand D., Groupes algébriques linéaires et théorie de Galois différentielle, Cours de troisième cycle, Université Paris VI, 1986.

[5] BIRKHOFF G.D., The generalized Riemann problem for linear differential equations and the allied problems for linear difference and $q$-difference equations, Proc. Amer. Acad. 49 (1913) 521-568.

[6] Borel A., Linear Algebraic Groups, 2nd Edition, Springer-Verlag, 1991.

[7] CAno J., Ramis J.-P., Théorie de Galois différentielle, 1999, in preparation.

[8] Chevalley C., Introduction to the Theory of Algebraic Functions of One Variable, in: Mathematical Surveys, vol. 6, American Mathematical Society, Providence, RI, 1963.

[9] Cohen R., Difference Algebra, Interscience Press, 1965.

[10] Deligne P., Équations différentielles à points singuliers réguliers, in: Lecture Notes in Mathematics, vol. 163, Springer-Verlag, 1970.

[11] Deligne P., Catégories tannakiennes, in: Cartier et al. (Eds.), in: Grothendieck Festschrift, vol. II, Birkhäuser, 1990.

[12] Deligne P., Milne J., Tannakian categories, in: Deligne, et al. (Eds.), Hodge Cycles, Motives and Shimura Varieties, in: Lecture Notes in Mathematics, vol. 900, Springer-Verlag, 1989.

[13] Di Vizio L., Arithmetic theory of $q$-difference equations. The $q$-analogue of Grothendieck-Katz conjecture on $p$-curvatures, Prépublication de l'Institut de Mathématiques de Jussieu, no 286, 2000. Invent. Math., submitted for publication.

[14] ETINGOF P.I., Galois groups and connection matrices of $q$-difference equations, Electronic Research Announcements of the AMS 1 (1) (1995).

[15] Gasper G., Rahman M., Basic Hypergeometric Series, in: Encyclopedia of Mathematics, vol. 35, Cambridge University Press, 1990.

[16] HENDRIKS P.A., Algebraic aspects of linear differential and difference equations, Thesis, University of Groningen, 1996.

[17] InCE E.L., Ordinary Differential Equations, Dover Publications, 1956.

[18] Katz N.M., On the calculation of some differential Galois groups, Invent. Math. 87 (1987) 13-61.

[19] Praagman C., The formal classification of linear difference equations, Proc. Kon. Ned. Ac. Wet. Ser. A 86 (1983).

[20] VAN DER Put M., Singer M.F., Galois Theory of Difference Equations, in: Lecture Notes in Mathematics, vol. 1666, Springer-Verlag, 1997.

[21] Ramanujan S., Collected Works, Chelsea, 1927.

[22] RAMIs J.-P., About the growth of entire functions solutions to linear algebraic $q$-difference equations, Annales de Fac. des Sciences de Toulouse Sér. 6 I (1) (1992) 53-94. 
[23] Ramis J.-P., Fonctions $\theta$ et équations aux $q$-différences, Unpublished notes, Strasbourg, 1990.

[24] Ramis J.-P., About the Inverse Problem in Differential Galois Theory. The Differential Abhyankar Conjecture, 1999, in preparation. A summary appears in:

BRAAKSMA B.L.J., IMminK G.K., VAN DER PUT M., The Stokes Phenomenon and Hilbert's 16th Problem, World Scientific, 1996.

[25] RAMIS J.-P., SAULOY J., ZHANG C., Local analytic classification of irregular $q$-difference equations, 2001 , in preparation.

[26] Sauloy J., Théorie de Galois des équations aux $q$-différences fuchsiennes, Thèse, Université Paul Sabatier, Toulouse, 1999.

[27] SAUlOY J., Systèmes aux $q$-différences singuliers réguliers : classification, matrice de connexion et monodromie, Annales de l'Institut Fourier 50 (4) (2000) 1021-1071.

[28] Sauloy J., La filtration canonique par les pentes d'un module aux $q$-différences, C. R. Acad. Sci. Paris (janvier 2002).

[29] SAULOY J., La filtration canonique par les pentes des modules aux $q$-différences et le gradué associé, 2002 , in preparation.

[30] SAULOY J., Local Galois theory of irregular $q$-difference equations, 2002, in preparation.

[31] SAULOY J., Galois theory of fuchsian $q$-difference equations, Long version of the present paper, 2002, url: picard.ups-tlse.fr/ sauloy.

[32] SAULOY J., La filtration canonique par les pentes des modules aux $q$-différences et le gradué associé, Rédaction d'exposés au Groupe de Travail "Équations aux q-différences", 2001, url: picard.upstlse.fr/ sauloy.

[33] SERRE J.-P., Groupes algébriques et corps de classes, Hermann, 1959.

[34] Seshadri C.S., Fibrés vectoriels sur les courbes algébriques, in: Astérisque, vol. 96, Société Mathématique de France, 1982.

[35] SpRINGER T.A., Linear Algebraic Groups, 2nd Edition, Birkhäuser, 1998.

[36] WAsow W., Asymptotic Expansions for Ordinary Differential Equations, Dover Publications, 1965.

[37] WeIL A., Généralisation des fonctions abéliennes, J. Math. Pures Appl. 17 (1938) 47-87.

[38] ZHANG C., Une sommation discrète pour des équations aux $q$-différences linéaires et à coefficients analytiques: théorie générale et exemples, in: Proceedings of the Workshop "Differential Equations and Stokes Phenomenon", Groningen, 2001, also as a preprint of the Université Paul Sabatier, Toulouse.

\footnotetext{
Jacques SAULOY

Laboratoire Émile Picard, UMR 5580,

Université Paul Sabatier,

118 , route de Narbonne,

31062, Toulouse Cedex, France

E-mail: sauloy@picard.ups-tlse.fr
} 